Hotchkiss, Neil

Flora of the Patuxent Research

Refuge, Maryland 

1 



\section{FLORA of the}

Patuxent Research Refuge<smiles>C[Al][Al][Mg]</smiles>
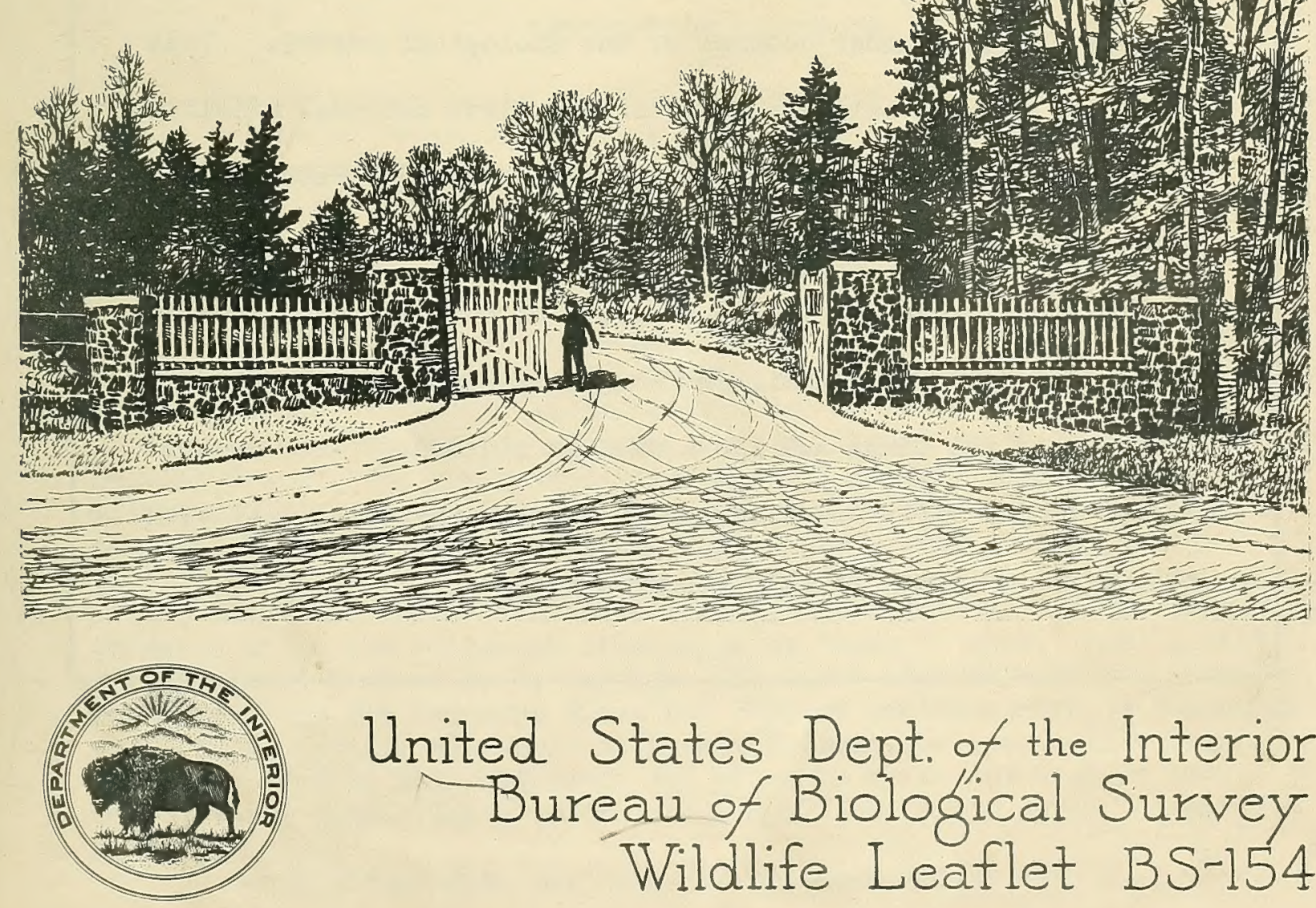

United States Dept. of the Interior
Bureau of Biolofical Survey
Wildlife Leaflet BS-154 


\section{LIBRARY \\ THE NEW YORK BOTANICAL GARDEN BRONX, NEW YORK 10458}

\section{FOREWORD}

Recognizing the complicated interrelationships of wildlife with man's changing uses of the land, the Biological Survey has for many years conducted studies in various parts of North Amerm ica, obtaining facts on which to base wise management of our wildife resources. Opportunity for research has been lacking on lands where deliberate changes in management practices and where the habitat requirements of the native species of wild animals could be under control of the Biologioal Survey. This gave Iise to the establishment of the first national wildlife experiment station of a general nature. On the Patuxent Research Refuge, wildiffe problems relating to the production of agricultural crops and to the practice of forestry will receive careful study. As a basis for longutime research the physiography and the fauna and flore will be studied. This contribution is the first of a number of reports that will deal with the natura. history of this research refuge. 


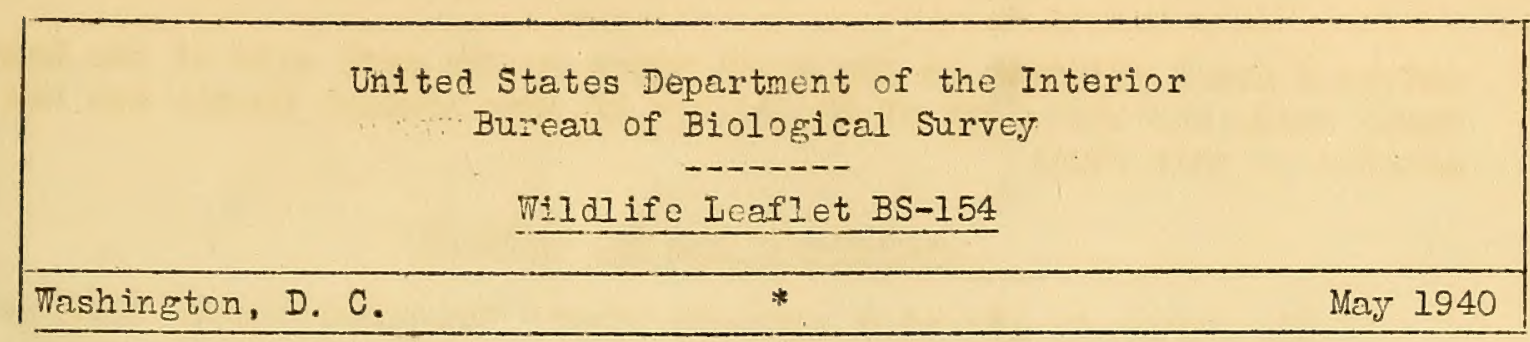

FLORA OF THE PATUXENT RESEHARCH RERUGT, MARYLAND I/

By Neil Hotchkiss, Biologist, Section of Food Habits DIvisicn of Mildlife Research

\section{Contents}

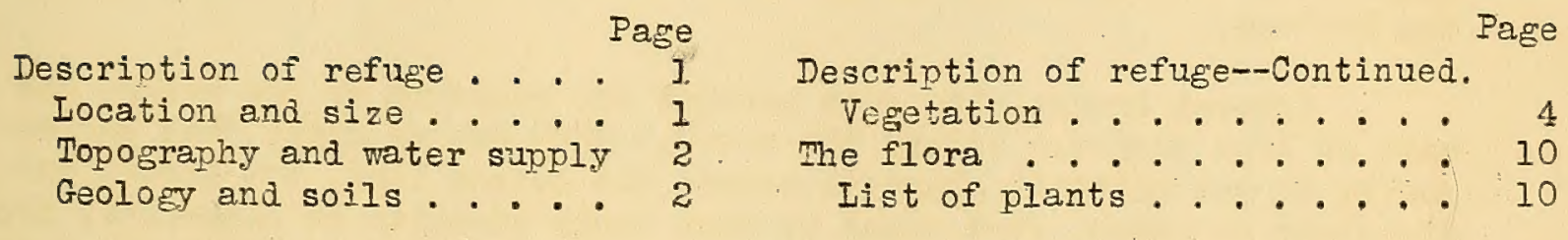

\section{DESCRIPTION OF REFUGE}

The folloving notes are based on observations and collections made by the writer on 78 nonconsecutive days, scattered through the growing and fruiting seasons from October 19, 1936, to July 25, 1939, on the Fatuxent Research Refuge, maintained by the Bureau of Biological Survey.

\section{Location and Size}

The Patuxent Research Refuge lies along both sides of the Patuxent River in Prince Georges and Anne Arvndel Counties, Md., from about 3 miles south-southeast of Iaurel southeastward for approximately 3 miles in a strip varying from $3 / 4$ to $1-3 / 4$ miles wide; thence southwesward, in Prince Georges County, for approximately 3 miles in a strip varying from $1 / 4$ to 1 mile wide, to a point about $1-1 / 2$ miles west of Bowie. (See map, p. 3; also the U. S. Geological Survey's map of the Iaurel quadrangle.)

There are three seperately fenced, but adjacent, tracts, which are referred to in the following aiscussion as "west, " "east," and "soutin" (in the plant list as $\mathbb{T}, \mathbb{E}$, and S.). The first lies between the IaurelBowie road and the Fatuxent River and extends southeastward to Telegraph Road, which crosses the River at Duvall. Bridge; the second lies across Telegraph Road to the southeast; the third is south and west of the Laurel-Bowie road. The 3 tracts total more than 2,000 acres. A small. 
unfenced iract adjacent to the south tract on the east side of the LaurelBowie road, and that part of the refuge in Anne Arundel County are not covered in this stuây.

\section{Zopography and Water Supply}

The refuge is situated entirely within the watershed of the Patuxent River, which here flows throwgh a valiey the maximum dimensions of which are about 3 miles wide and 750 feet deep. The altitude of the area varies from less than 80 feet above sea level along the river to more than 220 feet ncar the west anà south ends, but the slopes are mostly gentle, except for frequent 5- to 15-foot binffs along the highland side of the bottoms. The river falls 20 to 25 feet in its rather croolred course through a part of the refuge, traversing for the entire distance a bottom land that, on the southwest side of the river, except at ore place, is a quarter to half a mile wide. In places, between the bottoms and the upland slopes, are broad, nearly level terraces that lie only a few feet above the bottoms.

Several brooks rise just outside the boundary of the refuge and fiow more or less directly toward the river. They are so small that the majority do not maintain channels across the bottom land, but spread out or disappear on broad, flat areas or lose their identity in high-water channels along the highland side of the bottoms. One and one-half miles is the greatest length of any brook except the southeasternmost, Cash 3ranch, which drains the south tract and has a length of over 3 miles. Its 1 ower course is outside the refuge boundary. Cash Branch has been dammed to form 2 lakes in the south tract. In most years, the bottom lands are flooded on one or nore occasions for a few days at a time, but the river nomally flows between well-defined banks. A small flow of water continues through a fer shollow side channels, except in sumer; and in places along: the highlend side of the bottoms there are pemanent swanpy areas. The deepest and largest of these, which is more of a peat bog than a river swamp, lies just southwest of Duvall Bridge. A few springs occur along the bottom-land bluifs and elsewhere.

\section{Geology and Soils}

The refuge is only a few miles from the inner boundary of the Atlantic Coastal Plain, which follows a northeast-southwest line in the vicinity of Iaurcl. Underlying strata are all unconsolidated sediments of Cretaceous age.

The soils heve developed mostly fron weathered Coastal Flain rocks. An exception is the Congaree silt loom (See 1925 Soil Survey of Prince Georges County, Md.), wich covers the Patuxent bcttom land but which hes been washed there from the Piednont Platear. This loam is potentially one of the most productive of soils. Its origin partially accounts for this and for the fact that on the better drained perts of the bottom land many "rich woods" species of herbaceous flomering plants occur that are characteristic of the Piedront Flateau and that have not been discovered 


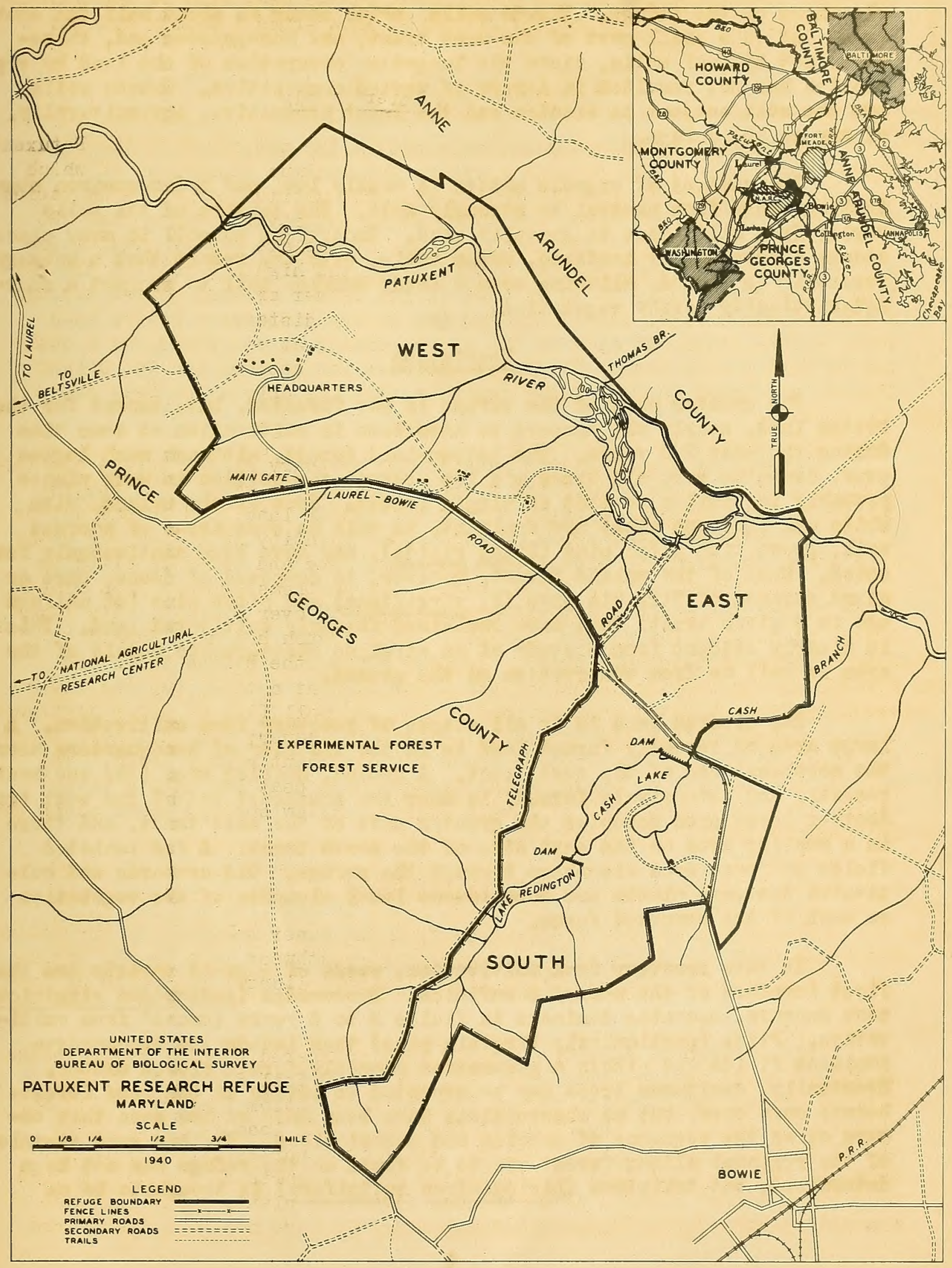


elsewhere on the refuge. Tuxedo soils, which occur on about half the south tract and on a small part of the west tract, are homogeneous and, therefore, not typical soils, since the long-time occupation of the land by vegetation has not resulted in laycrs of varied composition. Tuxedo soils are the most subject to erosion and the ieast productive, asriculturally, of any on the refuge.

The content of organic matter is mostly low, and hydrogen-iron concentrations range from neutral to strongly acid. The texture of the soils varies from silt loans to gravelly sand. Irpervious subsoil in many places causes a perched, or confined, water tobie, with the result that a subsurface reservoir is formed, allowing even a sandy surface soil to support a characteristically marshy vesetation.

\section{Tegetation}

The greater part of the refuge is now forested, but, except for the bottom land, nearl- all appears to have been in cultivation at some time during the vost 200 years. The botton-iand forest, el though much 10 gged over, contains some old trees ardits present composition in many places probably is similar to that of virgin forest. Some of the upland, with white oak (Grercus alba) now dominant, as mell as some areas of poorest soil, grown in pitch pine (Pinus rigida), may have been continuously forested. Wuch of the upland forest, homever, is composed of cense, pure or mixed strands of Virginia pine ( $F$. virginiana) and pitch pine (of uniform age in a given tract) that have reclaimed formerly cultivated land. This is clearly evident from a study of an airplene photographic mosaic of the area as weli as from observetion on the ground.

The cleared land is in all stages of recovery from cultivation. A large area of recently farmed lend is in the vicinity of headquarters near the northwest end of the vest tract. A second, sneller aree, (the one most recently and intensively farmed) is near the southeast end of the rest tract. Another lorge area occupies the greater part of the east tract, and there is a smaller area on the east side of the south tract. A few isolated fields are scattered elsewhere through the refuge. Old orcharas and cultivated dooryard plants are conspicuous local elements of the vegetation on each of the unmorked farms.

In this recovery from cultivation, weeds of a great variety are the first invaders of the unused cornfields. Broomsedge (Andropogon virginicus) soon appears, becoming dominant in fields 3 to 5 years removed from cultivation. Pines (particularly Virginia pine) then invade the broomsedgedominant fields and within a generation commonly form a closed canopy. Eventually, deciduous trees may be expected to invade as the pine forests become more open, but no observations have been made on how soon this occurs or on the sequence of species and associations. Thether good examples of the regional climax forest are to be found on the refuge has not been determined, but tuliptree (Iiriodendron tulipifera) is thought to be an 
indicator here of a stage of vegetational development apnroximating the climax.

On formerly cultivated damp soils, sedges, rushes, grasses, and other native herbs quickly form a dense cover. This, in turn, is frequently invaded by sweetgm (Iiquidambar styceciflua), winich locally forms pure stands as dense as the pines on well-drained sites. Further stages in this line of succession have not been studied.

Plant commities are of numerous and often complex types, because of the many. variations in soil, sites, and other environmental factors, and these are further complicated by man's dresijc disturbance of the original vegetation. Their exact definition and their relationships to each other remain to be investigated. A brief description, however, will indicate the dominant vegetation, grouped in 10 plant communites, as follows: (1) .water; (2) marsh; (3) wet meadow; (4) shrub swamp; (5) swamp forest; (6) bottom-land forest; (7) terrace forest; (8) slope and upland forest; (9) abandoned fields; and (10) hedgerows and poods margins.

1. Water.--Aquatic plants are rare on the refuge, because of the heavy shading of most of the waters by trees, the swiftness of the river and the brooles, and the impermanence of water in the shallow bottom-land runs. The species that have been found are sufficiently interesting as indicators of solt- or acid-mater conditions to be listed as follows:

Potamogeton epihydrus--river.

P. pusillus--brook.

Heterenthere reniformis--pond.

Podostemum ceratophyllum--river.
Callitriche heterophylla--springs and runs.

Utricularia geminiscapa--brook.

2. Warsh. - The areas in marsh are small but widely scattered. Wiarsh plants occur clong narrow bottom-land runs for short distances, in and along the brooks, and in openings in and along the margins of shrub siamps. The cominonest species are:

Isoetes engelmanni

Sparganium spp.

Sagittaria pubescens.

Echinochloa crusgalli.

Ieersia oryzoides.

Carex lupulina.

C. typhina.

Eleocharis obtusa.

Juncus spp.

Polygonum arifolium.

P. hydropiperoides.

P. bunctatum.
Polygonum sagittatum.

Nuphar advena.

Renunculus laxicaulis.

R. pusillus.

Hypericum mutilum.

H. virginicun.

Viola lanceolata.

Iudvigia palustris.

Proserpinaca palustris.

Lycopus spp.

Bidens connate. 
3. Tet meadow.-Many acres of formerly cultivated fields scattered throughout the refuge are nearly level and have a compact clay subsoil, resulting in a confined water tabie and enough moisture in the surface soil to maintain a great variety of typically marshmendow plants. The growth is much the same whether the fielos are on terraces just above the level of the river bottoms or on the broad tops of the higher hills. The cominonest olants are:

\author{
Panicum microcarpon. \\ Carex Iurida. \\ Eleocharis capitata. \\ Rhynchospora capitellata. \\ Juncus spp. \\ Tiola sagittata.
}

Rhexia spp. Oenothera Iongipedicellata. Apocynum cannabinum, var. pubescens. Pycnanthemum spp. Eupatorium spp.

Iiquidamoar styraciflue is an earty and abundant invader of wet meadows. Where wet-meadom soil has recently been bared, Cyperus strigosus and Fimbristylis mucronuleta are common.

4. Shmb stamp.--The largest shrub stramp is on the highland side of the bottoms near the southenst end of the rest trict; other much smaller ones are scattered along the higinand side of the bottoms, along the valleys of the brooks, on the river-valley terraces, and rarely in seepage areas on the slopes. The most abundant shrubs and the conspicuous herbs are:

\section{Shrubs}

Alnus rugosa. Magnolia virginiana. Aronia arbutifolia. Rosa parustris. Ioxicodendron vernix.
Ilex verticillata.

Clethra alnifolia. Rhododendron viscosum. Vacciniurn atrococcum. Cepralanthus occidentalis.

Herbs

Oswunda cinnamomea.

Carex Iaevivaginata.

Carex folliculata.

C. stricta.

5. Sramp forest.--Small tracts of swamp forest occur along the highland side of the bottoms, on the terraces, along brooks, and in upland hollows. Douinant trees ana common shrubs and herbs are:

\section{Trees}

Betula nigra.

Quercus palustris.

Q. phellos.

Magnolia virginiana.
Iiquidambar stryraciflua. Acen rubrum.

Iyssa sylvatica. 


\section{Shrubs}

Similax spp.

Ilex verticillata.

Ievcothoe racemosa.
Lyonia Iigustrina.

Viburnum nudum.

V. püoscens.

\section{Eerbs}

Trooảwardia areolata.

Carex crinita.

Csmunda cinnamoniea.

C. folliculata.

Cimna arundinacea.

Symplocarpus foetidus.

Glyceria striata.

6. Bottor-land forest.--Forest trees cover nearly all the extensive bottom lands of the west and east iracts. STight differences in elevation account for striking differences in the flore of localities only. $\mathrm{A}$. f feet apart. The herbaceous vegetation is particularly luxuriant and includes practically all of the "spring flowerg" of the refuge. The dominant trees and common shrubs and herbs are:

\section{Trees}

Betula nigra.

Carpinus caroliniana.

Fagis grandifolia.

Quercus palustis.

Q. prinus.

UImus americana.

Shmibs

Iindera aestivalis. Toxicodendron radicans.

Herbs

Cinna arundinacea. Festuca obtusa. Poa sylvestris. Ari scema triphyjlum. Ery uhronium americanum. Uvuraria sessilifolia. Boenimeria cyindrica. Laportea canacensis. Polvgonum virginianum. Stellaria longifolia. Claytonia virginica. Ranunculus a.oortivus.
Iitiodendron tulipifera.

Iiquidambar styraciflua.

Cornus florida.

Fraxinus spp.

Viburnom prunifolium.

Evonymus americanus.

Renunculus recurvatus.

Podophyllum peltatum.

Dentaria laciniata.

Geun canadense.

Ampinicarpa bracteata.

Viola affinis.

Panax trisolium.

Cryptotaenia conadensis.

Steironena ciliatun.

ivimulus aletus.

Gelium aparine.

Lobelia cardinalis. 
7. Temrace forest.-- This is not an outstanding type. In generaI it resembies that of the slopes and uplands except that, being level, it retains nore moistrire and tis is reflected in sit git itferences in the vegetation. Wuch the same troe of lorest occurs in piaces a'ong the bottom-land bluffs and on the gentle slopes tordering the valieys of some of the brooks. Terrace forest appars to be the aearest approsin to a ciimax of any trpe of vegetation ooserved on the refuge. Species comoner here than elscwhere are Farus Erandifolta cnd Iiriodenarou tulipifera. Ilex opaca and Zalmia latifolia are conmon members of the underetory. dubunant heros are:
Dryopteris noveboracensis.
Medecia viiginiana.
Iycopodium complanatur.
Carer stanii.
\#pifagus virziniana.
iritchella repens.

E. Slope and 1npland fcrest.--This cheracterizes a greater part of the erea than docs any other type. It is usueliy of mixed oak and pine, but on recently cleared land it is pure pine. Or some cut-over areas the stand is largely ock. The domirant trees and characteristic shruos and herbs of slcoe and upiand forest are:

\section{Trees}

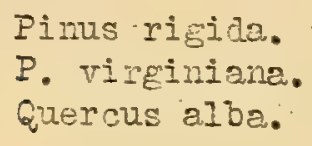

Quercus coccinea.

Q. falcats.

Q. velutina.

Shrubs

Kalmia Latifolia.

Bhojodenaion nuiflorum.

Vaccinium stamineum.

v. vecillars.

Eerbs

Pyrola amezican.

Epifc.gus virzinicna.

Pteridium Iatiusculum.

Chimaphila maculata.

9. Abandonea fields.--Recantly cultivatca land comprises a large acreage, 0 stiy on slopes and uplanas, but also to sone extent on terraces along the Patuxent Valley. The soils of the latt:r are noticeably more scmäy than elsewhere, cind this is reflected in differences in vegetation. A characteristic of fields that have been out of cultivation for 1 to 3 jears is the great variety of weedy species that grom tincre. Lost common of these are: 
Aristida dichotoma.

A. Iongespica.

Bromus commutatus.

Panicun lindheimeri.

Paspalum spp.

Rumex acetosella.

Rubus sp. (dewberry).

Cassia fasciculata.

C. nictitans.

Iespedeza striata.

Irifolium spp.

Polygala curtissi.

Hypericum gentianoides.
Trichostema dichotomum.

Solanum carolinense.

Iinaria canadensis.

Plantago lanceolata.

Diodia teres.

Specularia perfoliata.

Achillea millefolium.

Ambrosia artemisifolia.

Anthemis arvensis.

Chrysonthemum leucanthemum.

Erigeron spp.

rrigia virginica.

Meadows and pastures are usually weedy. A few species that appear to be somewhat more characteristic of these than of abandoned cultivated fields are Triodia flava, Remincilus bulbosus, and Plantago rugelii. Plants that do not occur elsewhere generally but that are conspicuously abundant on the sandy terrace soils of the west and east tracts are:

Eragrostis spectabilis.

Smilax glauca.

Potentilla canadensis.

Desmodium spo.
Iespedeza spp.

Iechea racemulosa.

Tecoma radicans.

Solidago juncea.

Tithin 2 or 3 years after a field has been abandoned, broomsedge (Andropogon virginicus) begins to appear. It soon becomes dominant and maintains dominance until it $\dot{s}$ crowded out by invasion of pines. Pinus virginiana and $P$. rigide both imvade broomsedge fields, but the former is nearly everywhere in much greater abundance. Sassafras officinale is also found in old fields, commly appearing in the poorer soils soon after the fields have been abandoned. On sterile slopes and hilltops, bluestem (Andropogon scoparius) is sometimes dominant. These areas appear to have been cleared for a much longer time and they are changing much more slowly than are the more typical older fields. Comon species associated with stands of bluestem are:

\section{Desmodium spp. \\ Iespedeza spp. \\ Gerardia spp.}

Chrysopsis mariana.

sericocarpus linifolius.

10. Hedgerows and woods margins.--Many of the fields are bordered by hedgerovis dominated by a variety of trees having dense undergrorth of shrubs and woody vines, which, likewise, in many places form a dense barrier along the edges of the roods. Among the most conspicuous species are:

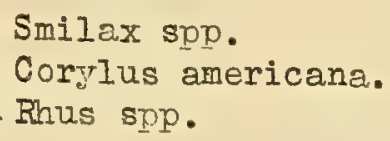

Smilax spp.

Corylus americana.

Rhus spp.

Vitis spp.

Clethra alnifolia. Iyonia ligustrina. 
Seven hundred and six species of flowering plants and ferns have been collected on the Patuxent Research Refuge by the writer, including 93 grasses, 31 sedges, 79 composites, 46 trees, and 55 shrubs and woody vines. All but 7 of the species are included in Hitchoock and Standley's 1919 Flora of the District of Columbia and Vicinity (or its 1930 Supplement by icitee), which describes a total of 1771 species. This is a rich flora considering that so many plants of the Washington region are restricted to the Piedmont Plateau or to the Potomac River Valley.

A number of species listed in the "District Flora" as occurring only on the Piedmont Plnteau have been found to be common on and in the vicinity of the Patuxent bottoms. The Piedmont-derived soil ind the contimity of the bottom lands froin the lower Piedmont Plateau through the Coastal Plain help to explain this distribution. Although Sanguinaria canadensis has been identified from growing plants on the refuge, it is the only species in the following list that is not yet represented by one or more permanently filed specimens. Possibly 100 additional species could be listed in 2 or 3 more years of collecting, but there are not many common plants, with the exception of certain composites, carices, and panicums, that have been overlooked.

\section{Iist of Plants}

"West," "east," and "south" (W, $\mathbb{E}$, and $S$, respectively), refer to the three separately fenced tracts of the refuge. Numbers in parentheses, except. as otherwise noted, refer to plants collected by the writer; identifications other than those by the writer are so indicated.

\section{POLYPODIACEAE:}

Aspleniurn platyneuron (I.) Oakes.--Occasional; $W, \mathrm{~s}$; hedgerows and former clearings. (5155)

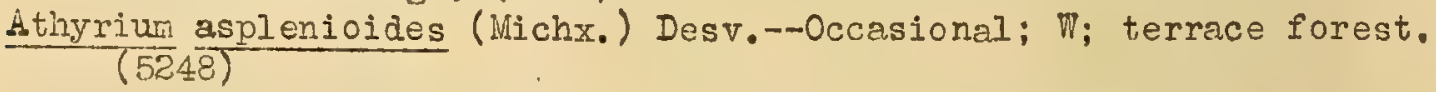

Dennsteedtia punctilobula (Michx.) Moore.--Occasional; W, S; mostly terrace forest. (5438)

Dryopteris cristata (I.) A. Gray.--Occasional; W; swamp forest. (5055)

D. hexagonoptera (Michx.) C. Chr.--Occasional; W; well-drained bottomland forest. (5310)

D. marginalis (I.) A. Gray.-Rare; W, S; terrace and slope forest. $(5054)$

D. noveboracensis (I.) A. Gray.--Common; $W$, s; terrace forest. (5423)

D. Spinulosa (!uel1.) Kuntze, var. intermedia. (Muhl.) Underw.--Occa sional; Ti; terrace forest. (5056, det. W. R. Maxon)

D. thelypteris (I.) A. Gray.--Occasional; W, E, S; open shrub swamp.

Onoclea sensibilis I.--Occasional; W. E; wet meadow. (5586)

Polystichum acrostichoides (Michx.) Schott.-Occasional; $\mathbb{W}, \mathbb{E}, \mathrm{S}$; terrace and slope forest. (5445)

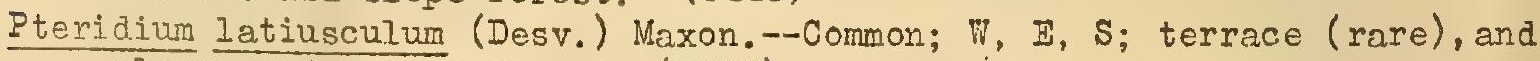
slope and upland forest. (5608)

Toodwardia areolata (I.) Moore.-Common; $\$, E, S$; swamp forest. (5500)

W. virginica (I.) Sm.--Rare; W; shrub swamp. (5503) 
OSMUNDACEAE:

Osmunda cinnamomea I.-Common; $\mathbb{W}$; shrub swamp and swamp forest. (5193) O. regalis I. Var $_{\text {forest. }}$ sloectabilis (Mill.d.) Gray.-Occasional; N; swamp OPHIOGLOSSACPAE:

Botrychium dissectum Spreng.--Rare; W, S; former clearings, and swamp forest. (5663)

B.'dissectum, form a obliquun (WhI.) Fern.--Occasional; $\mathbb{W}$, S; wet meadow, swamp forest, and hedgerows (5053, 6026)

B. virginianum (I.) Sw.--Occasional; W; well-drained bottom-land forest. $(5177)$

\section{IYCOPODIACEAE:}

Iycopodium clavatum I.--Rare; S; woods margin. (5794)

I. complanatum I., var. flabell forre Fern.-Abundant; $\mathbb{W}, \mathrm{S}$; terrace and slope forest. (5444)

I. Iucidulun Michx. -Occasional; ii; terrace forest. (5048)

I. obscuram I. -Conmon; $W$, S; terrace forest. (5049)

SEILAGINELIACEAT:

Selrginella apoda (I.) Torn.--Occasional; $\pi, E$; wet meadow and open swamp forest. (5130)

ISOETACEAE :

Isoetes engelmanni A. Br.-Occas: onal; $\mathbb{T}$; runs in bottom-land forest. (5175?, 5909)

PINACELE:

Juniperus virginiana I.-Occasional; $\mathrm{W}, \mathrm{S}$; bottom-land to upland forest. (5768)

Pinus echinata Mill.--Rare; $\pi$; slope forest. (5805)

P. rigida Mill. - Abundant; $W$, $S$; terrace forest (rare), slope and upland forest and abandoned fields. (5051, 5803).

P. strobus I.--One tree; S; upland forest. (5144)

P. taeda I. -Rare; S; woods margin. (5744)

P. Virginiana Mill.--Very abundant; $\mathbb{W}, \mathrm{s}$; terrace forest (rare), slope and upland forest and abrndoned fields. (5052)

TYPHACEAE:

Typha latifolia I.--Rare; W, E, S; marsh and open shrub swamp. $(5283,6029)$

SPARGANIACEAE:

Sparganium americanum Nutt.-Occasional; S; marsh. Relative abundance of the two species has not been determined. (6328)

S. andirocladum (Engelm.) Morong. (S. Iucidum)-Occasional; $\mathbb{W}$, s; marsh. (5753, 5328 ?, $6030^{-}$?)

\section{NAJADACEAE:}

Potamogeton epihydrus Raf.--Rare; $\pi$, $\mathbb{F}$; margin of river. (5491)

P. pusilius I., var. typicus-Rare; s; pond below dam. (6077) 
AIISMACEAE:

Alisma plantage-aquatica I.-Occasional; T, E, S; marsh. (5316) Sagittaria latifolia Willd.--Occasional; W, E; marsh. (6015) S. pubescens líuhI. - Occasional; W, S (comon along Cash Branch); marsh. (5329)

GRAMIIEA (Determined by J. F, Swallen, except as otherwise noted): Agropyron repens (I.) Beauv.--Occasional; F; abandoned fields. (5772, 5893)

Agrostis alba I.--Iocally common; W; wet meadow. (5272)

A. hiemalis (Walt.) B. S. P.--Occasional; $T$, E; wet meadow. (5216)

A. perennans (Walt.) Tuckerm,-Occasional.; W; terrace and slope and upland forest. (5474)

Aira caryorhyllea $I .--O c c a s i o n a l ; \pi, \mathbb{E}, \mathbf{S}$; roadsides and abandoned iields. (5145)

Andropogon elliottii Chapm.-Occasional; $T, \mathrm{I}, \mathrm{S}$; abandoned fields. $(5739)$

A. scoparius Micht.--Iocally abundant; $\mathbf{T}, \mathrm{E}, \mathrm{S}$; abandoned fields. (5591)

A. Virginicus I.--Very abundant; $\mathbb{T}, \mathbb{E}$; abandoned fields. (5649) Anthoxanthur odoratum I.-Occasional; $T$; abandoned fields. (5199) Aristida dichotoma Michx.--Common; 7,5 ; abandoned fields. (5745) A. Ionoespica Poir.-Common; T, E; abandoned fields. (5559, 5629)

A. oligantha Michx.--Rere; $\Pi$, S; abandoned fields. (5785)

A. purpurascens Poir.--Rare; $\$$; abandoned field on sandy terrace. (5650)

Braclyelytrum erectum (Scinreb.) Beauv.--One plant; $\%$; bottom-land forest. (5489)

Bromus commutatus Schrad.-Common; W; abandoned fields. (5226)

Calamagrostis cinnoides (Mhl.) Barton.--Occasional; W, S; wet meadow (5587)

Cenchrus pauciflorus Benth.--Occasional; $\mathbb{T}, \mathbb{E}$; abandoned fields. (6018)

Cinna arundinacea I.--Abundant; $W, s ;$ swamp and bottom-land forest. (5053 1/2, 5603)

Cynodor dactylon (I.) Pers.-Occasional; S; in gravel road. (6324)

Dactylis glomerata L.-Occasional; T; margin of old road. (516I)

Danthonir spicata (I.) Beruv.-Common; W; abandoned fields. (?) (5237)

Digitaria filiformis (I.) Koel.--Occasional; T, S; along wood roads and in recent clearings. (5706)

D. ischaemum (Schreb.) Muhl.-Iocally comon; $\pi$, $\mathbb{E}$; roadsides and abandoned fields. (5510)

D. sanguinalis (I.) Scop.--Occasional; T; yards and abandoned fields. (5505)

Echinochloa crusgalli (I.) Beauv.-Occasional; $\nabla$; marsh and wet meadow. (5477, 5764, 6103)

Fleusine indica (I.) Gaertn.-Occasional; E; roadside. (5383)

Elizms virsinicus I.--Occasional; $\pi$; bottom-land forest. (5487)

Eragrostis capiliaris (I.) Nees.-Rare; 7 ; recent clearing. (5777)

E. cilianensis (All.) Link.-Occasional; 7 ; yards and abandoned fields. (5504) 
GRAMINEAT--Contined.

E. hypnoides (Iam.) B. S. P.--OccasionaI; W, S; wet meadow. (6089)

I. Xectinaced (Michr.) Neas.--Erre; $T$; in old road. (5693)

E. spactabilis (Fursh) Stud.--occasional; 7 . E; abandonea fields on sandy terraces. (5531)

Festuca elatior I.--Rare; E; yard. (5902)

F. miros I.-Occasional; $\Pi$, $\Xi$; abandoned fields. (5848, det. A. Chase)

E. obtuse Spreng.-Common; $\pi$; bottor-land forest. (5488)

F. Octoflora Talt.--Occasional; $\pi, \mathbb{E}$; woods margins and abandoned fields. (5213)

Glvceria canadensis (Michx.) Trin.--Rare; S; marsh at south end of lake. (6113, 6327)

G. obtusa (Muhl:) Trin.--Locally common; s; marsh. (5568)

G. Dallida (Torr.) Trin.--Rare; $\pi$; marsh in botton-land runs. (5898)

G. seotentrionalis Hitchc.-Occasional; T, J. S; marsh in bottom

land runs and in swamp forest (rare). (5181)

G. Striate (Iam.) Eitchc.-Comon; $W_{1}, \pm, S$; stamp forest. (5203, 5284)

Folcus lanatus I.-Occasional; $\%$; margin of old road. (5150)

Eystrix patula Moench. - Rare; T; bottom-land forest. (5490)

Leersia oryzoides (I.) Startz--Common; $\pi$, 5 ; marsh. (5472)

I. Virminica Filld.--Occssionel; i; bottom-lmud (and terrace ?) forest. (5473)

Iolium multiflorum Ian.--Rare, T; Jard. (5835, det. A. Chase)

I. perenne I.--Rare; W; Jard. (5881, det. A. Chase)

Muhlenbergia mexicana (I.) Mrin.--Rare; $\overline{\text { Ti }}$ jard. (6108)

19. schreberi Gmel.-Occasional;, I; yards and abandoned fields.

(5718)

M. Sylvatica Torr.--Rare; W; bottom-land forest. (6079)

Fanicur agrostoides Spreng.--Rare; S; wet meadow. (5790)

P. ancens lichx.--Occasional; $T, E, S$; abandoned fields. (5434)

P. ashei Pearson.--Occasionel (?); $\mathbb{T}$; uplend forest. (5670)

F. Daroulatum Michr.--Occasional (?); S; cleared shrub swamp (5592)

P. boscii Foir.-Occasional; $\pi$; partly cleared terrace forest. (5425)

P. caniliare I.- One plant; T; abandoned orchard. (5709)

. Clandestimur I.-Comon;

P. columbianum Scribn. --Rare (?); $\pi$; upland forest. (5671)

P. conititetum Schult.--Occesional; $\Pi$; terrace, and slope and upland

forest. $(5405,5669)$

P. depauperatum Muhl.--Occasional; T; recently cleared upland forest. (5197)

P. dichotomiflorum Wichx.--Common; $\pi$, s; recently disturbed soil of wet meadoms and yards. (6091)

P. dichotomum I. - Occasional; $7, \mathbb{E} ;$ well-drained bottom-land to upland forest. (5354, 5672)

P. Eattingeri Nash.--Rore; $\pi$; former cornfield. (5722)

P. huachucee Ashe-Occesional; $\pi$, S; tet mendow and recently cleared slope forest. (5340, 6094)

P. Iindheimeri Nash.-Common; T, $\Xi, S(?)$; wet meadow and abandoned fields. (5276, 5337, 5387) 
GRAMINEAE--Continued.

P. 1ongifolium Torr.--Cocisional; T; vet mendow. (5394, 5478)

P. microcaruon Muhl.-Occasionil; $\pi$, S; wet meadow and cleared shrub swamp. (5214, 5499, 6092, 6095)

P. philadelnkicum Bernh.--Occasional; T; abandoned fields on terrace and vpland. (5560, 5786)

P. polyaithes Schult.-Occasionel; W; open bottom-land and terrace forest and abandoned fields. (52s?)

P. scoparium Lam. -Occasional; T; wet meadow. (5336)

P. Sphaerocaspon EII.--Rare; $E$; abandoned field on sandy terrace. (5648)

P. stipitatum Nash.--kare; W; wet meadow. (5581)

P. verrucosum Mhl.--Conmon; $W, S$; recently cleared shrub swamp and swamp Iorest. (5498)

P. virgatum I.--One clump; I; abandoned field. (5734)

Faspalun leeve Hichx.--Occasional; $T$, E; Tet meador. (5368)

P. Iongipilum Nash.--Rare ( $P$ ); T, E; abanconed fields. (5367, 5417)

P. pubescens Mhl.--Occasional; $W$, E; abondoned fields on sandy terraces. (5397, 6023)

P. setaceum Michx.-Rare (?); I; abandoned field on sandy terrace. (536)

Plileum pratonse I.--Occasional; $\mathbb{H}$; margin of old road. (5455)

Foa annua I.-Occasional; T; wet madow. (5096)

P. autumalis Murl.-Occasional; W; bottom-land forest. (5166)

P. compressa I. -Occasional; $W, \mathrm{~s}$; abandoned fields. (5428)

P. pratensis I.--Occasional; T; margin or old roaa. (5151)

P. sylvestris A. Gray.-Common; W; bottom-land forest. (5131)

P. triviais I.-Occasional; $\bar{W}$; tottom-lend forest. (5169)

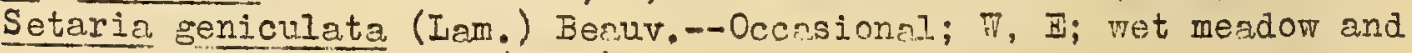
abandoined fields. (5584)

S. Iutescens (Weigel) E. T. Hubb.--Iocally comnon; T, E; abandoned fields. (5458)

S. viridis (I.) Beauv.--Occasional; $T$. abandoned fields. (5701)

Sorghastrum nutans (I.) Nash.-Occasional; $\mathrm{T}$; abandoned fields. (5580)

Sphenopholis intermedia (Eydb.) Hydb.--Occasional; T; bottom-land

forest. (5173)

Sporobolus voginiflorus (Torr.) Wood.--Iocally common; W; abandoned fields: $(5771)$

Triodia flava (I.) Sryth.-Common; $T, E, S$; abandoned fields. (5626)

Uniola laxe (I.) B. S. P.-Occasional; W; well-ärained bottom-land and terrace forest. (5484)

\section{CYPERACEAE:}

Bulbostylis capillaris (I.) C. B. Clarke.-Occasional; W, E, S; abandoned fields and recently cleared forest on sandy terraces. (5609)

Carex spp.--Determined by F. J. Eermann, except as otherwise noted.

6. rbscondita Mack.-Rare; W; terrace forest. (5252)

C. albolutescens Schwein.--Occasional; $\pi, E$; wet meadow. (5840,

C. annectens Bickn.--Occasional; W, E; margins of swamp forest. (5291)

C. bullatr Schkuhr.--Rare (?); $\mathbb{T}, S_{i}$ shrub swamp. (5793, det. Hotchkiss) (5880) 
CYP ARACEAE--Continued.

C. canescens I., var. disjuncta Pern.--Rare; W; open shrub swamp.

C. caroliniana Schw.-Occasionel; T; oper terrece forest and abandoned fields. (5260, 5302, 5873)

C. cephalophora Muhl.--Rare; $\mathbb{W}$; open terrace forest. (5872)

C. complanata Torr. \& Hook.-Cccasional; $:$; abandoned fields on sandy terraces. (5298, 5321)

C. convoluta Mack.--Rare; T; open terrace forest. (5874)

C. crinita Iam.-Common $\mathbb{W}, \bar{E}, \mathrm{~s}$; swamp forest. (5023, det. Hotchkiss) (5257)

C. debilis Michx.-Occasional; $\mathbb{T}$, $\mathbb{N}$; terrace forest. (5244)

C. digitalis Wilid.--Rare; T; open terrace forest. (5875)

C. emmonsil Dewey.--Rare; S; moist opening in upland forest. (5143)

C. festucacea Schkuhr.--Rare; $W$; open terrace forest. (5870)

C. Polliculata I.-Common; W, $\mathrm{E}, \mathrm{S}$; shmb swamp and swamp forest. (5246)

C. glaucodea Tuckerm.--Occasional; T, E, S; wet meadow and openings in terrace and upland forest. (5198, 5833, 5972)

C. hirsutella Mack.--Rare; T; abandonea field on sandy terrace. (5303)

‥ incomperta Bickn.-Occasionar; T, Đ; open shrub sramp. (5256, det. Fotchkiss) (5839)

C. intumescens Rudge.-Occasional; T. E; swamp and terrace forest. (5245)

C. Iacustris Willd.--Locally common; \#; margin of shrub swamp. (5882)

C. Iaevivaginata (Kitkenth.) Mack.--Comnon;, I; shrub swamp. (5227) (5838, det. Hotchkiss)

C. Iaxiflora Iam.--Occasional; W; open terrace forest. (5869)

C. Iongi ivack.--Rare; T; wet meadow. (F.J. Hermann 1037I)

C. Iupuliformis Sartw.--Rare; $\pi$; run in bottom-land forest. (6013)

C. Iupulina Whi.-Common; $\mathbb{T}$; marsh in bottom-land runs. (5766, det. lotchkiss) (5897)

C. luriaa Tahl.-Conimon; T, E; wet meadow. (5228, 5273)

C. richii (Fern.) Mack.--Occasional; $W$; open shrub swamp. (5255)

C. rosea Schluhr.--Rare; $\mathbb{7}$; terrace forest. (5247)

C. Scoperia Schkuhr.--Occssional; $T$, E; wet meador and shruo smamp. (5230, 5270) (5286, det. Hotchkiss)

C. seorsa E. C. Eowe.--Rare; T; margin of swamp forest. (5876)

C. Squarrosa I.-Occasional; $7, \mathrm{E}$; bottom-land forest. (5851, 5527)

(5928, det. Wotchkiss)

C. stipata Muhl.-Occasional; $\mathbb{W}, \mathbb{I}$; margin of shrub swamp and swamp forest. (5837) (5881, det. Eotchkiss)

C. Stricta Lam.--Common; $\mathbb{W}$, $\mathbf{E ;}$; shrub swamp. (5307) (5836 is var. strictior (Dewey) Carey)

C. Swanii (Fern.) Mack.-Common; T, E; swamp margins and terrace forest. (5251)

C. tribuloides Tahl.-Occasional; $\pi$; abandoned field along hedgerow and margin of swamp forest. (5908)

C. typhina Michx.--Occasional; $\pi, E$; marsh in bottom-land runs. (5028, det. Hotchkiss) (5357, 5765, 6019)

C. vulpinoidea Michx.--Rare; $\pi$; abandoned field. (5894) 
CYPERACEAE--Continued.

Cyperus crlinuricus (EIJ.) Brition--Occisional; $\mathrm{I}, \mathrm{S}$; recent clenrings in terrece anc mend forest. (5735)

C. esculentus I.--Rise; T; abnouned field. (6017)

E. filiculmis Vahl.-Occesional; I; ubundoned field on sandy terrace. (5360)

C. flavescens I.-Occastonn1; T, i; wet mèdor. (5564)

C. microdontus Torr.--Rore; W; wet neadow. (5565)

C. ovularis (Miahx.) Torr.--Occasional; ; ; wet meadow. (5338, 5583)

C. Fivalaris Kunth.--Occasional; T. E; Wet meador. (5067, 5563)

C. strigosus I.-Common; T, S; ret meadon. (5533)

Dulichium arundinaceum (I.) Britton.--Occasional W, S; marsh. (5573)

Fleocharis acicularis R. \& S. ( P)--Iocally common; W; pond. (5770)

E. capitate (I.) F. Er.-Common; T; Tet mendor. (5277)

E. obtusa (Willa.) Schult.--Common; $\mathbb{T}, \mathrm{s}$; marsh. (52,66, 5773, 6090)

Fimbilstylis mucronulata (Michr.) Blake.-Comon; $T$, I; ret neadon. $(5339,5362)$

Kyllinga pumile Micin.--Occasional; $\mathrm{iv}, \mathrm{S}$; met meadow. (5066, 5715) Rhyrchospora capitellata (Lichx.) Vahl.--Occasional; T; wet meadow. (5479)

R. corniculata (Iam.) A. Gray.--Rare; $\overline{\text {; }}$; marsh in jottom-land mans. (5027, 6012)

R. oymosa Ell.-..occasional; I; brushy wet meadow. (5376)

Scirpus atrovirens Mul.-Occasional; 7 ; clearing in swamp forest. (5290)

S. cyperinus (I.) Kunth.--Occasionai; 7, S; wet meadow. (6027)

S. debilis Pursh.--Jocally common; S; marsh. (5589, 5774, 6082)

S. eriophorum Michr.-Occasional; W; wet meadow. (5593, 5689)

S. polyohylus Tahl.--Occasional; $\pi$; clearing in swamp forest. (5327)

Scleria pauciflorc Mhhl.-Occasional; 1 ; orusiny wet meadow. (5377)

ARACEAT:

Ariscema triphylium (I.) Schott.-.Comon; $\pi$; bottom-land forest and shrub swamp. (5090, 5136)

Orontium aquaticum L.-Occasional; $\pi$, $\Xi$; runs in bottom-land forest. (5111)

Sumplocarpis foetidus (I.) Mutt.--IocalJy abundant; $\mathbb{F}, \mathbb{E}$; shrub swemp and stramp forest. (5811)

XYRIDACEAE:

Xyris caroliniona Walt.--Rare; $s$; sandy soil in cleared shrub swamp. $(5 \overline{574})$

S. torta J. E. Snith.-Occasional; T (?), E. S; wet mendom. (5372)

COMULIINATEAZ:

Commelina communis I.-Occasional; $\pi$; along road in terrace forest and margin of shrub stramp. (5404, 5468)

C. hirtella Vahl.--Rare;" W; margin of bottom-land man. (5451) 
PONTEDERIACDAE:

Heteranthera reniformis $R$. \& F.--Rare; $\pi$; pond. (5716)

Pontederia cordata I. --Kare; $\mathbb{E}$; warsh in bottom-land run. (5353)

JUNCACRAT:

Juncus acuminatus Michx.--Occesiona?; M; marsh. (5267)

J. Jufonius I.-Occasional; $W, \mathbb{I}$; moist seindy roadside. (5867)

J. canadensis J. Gay.-Occasional; T; wet meadow. (5595)

J. effusus I.-Common; $\mathbb{W}, \mathrm{I}, \mathrm{S}$; marsh and wet meadow. (527I)

J. mecer S. F. Gray.--Occasional; 7; vet meadow. (5269, 5274)

J. mar natus Rostk.-Occasional; $\boldsymbol{W}, \mathrm{S}$; wet neadow. (5268 and 5994, det. F. J. Hermann)

J. scirpoides Iam.--Occasional; $\mathbb{W}$; wet meadow. (5594)

J. secundus Beauv.-Occasional; W, S; wet meadow. (5993, det. $F$. J. Hermann)

J. tenuis Willd. (J. dichotomus El1.)-Occasional; $\mathbb{F}$; wet meadow. (‥J. Fermann I0366)

Iuzula echinata (Saall) Hernann.--Occasional; W; open terrace forest. (5105 aild 5871, det. F. J. Fermanu)

I. multiflora (Ihrh.) Iejeune, var. bulbosa (Wood) Hermann.--Occasional; $\mathbb{E}$; abandoned field on sandy terrace. (5809, det. F. J. Hermann)

IIIIACEAD:

Aletris farinosa I.-Occasional; $\mathbb{W}, \mathbb{s}$; wet meadow and sandy abandoned fields. (5304)

Allium canadense I.-Occasional; $T$; bottom-land forest. (5132)

A. Vineale I.-Iocally colimon; $\mathbb{W}$; abandoned fields. (5262)

Asparagus officinalis I.-Fiare; $\Pi, E$; abandoned fields. (5740)

Erythronium americanum Ker.--Comm; $\mathbb{N}, \mathbb{E}$; bottom-land forest. (5812)

Hemerocallis fulva I.-Occasional; $\mathbb{T}$; open forest and hedgerow. (6325)

Iilium superbur I.--Occasional; $\mathbb{W}$; wet meadow. (5395)

Maianthemuri canadense Desf.--Iocally comon; i; swamp forest. (5137)

Medeola virginiana I. - Common; $\pi$; terrace forest. (5138)

Ornithogalum umbellatum I.--Iocally abundant; 7 ; abandoned fields on sandy terraces. (5098)

Polygonatum oiflorum (Talt.) Ell.--Rare; 7 ; upland forest. (5664)

P. commutatum (R. \& S.) Dietr.-Occesional; $\mathbb{T}$, $\mathbb{E}$; bottom-land forest. (5i7l)

Smilacina racemosa (I.) Desf.--Occasional; $\mathbb{T}$; terrace forest. (5427)

Smilax glauce Walt.-Comon; $\mathbb{T}, \mathbb{E}, \mathrm{S}$; bottom-land to upland forest and abandoned fields on sandy terraces. (5762)

S. herbacen I.--Rare; $\mathbb{W}$; open terrace forest. (5604)

S. rotundifolia I.-Occasional; $\pi$; shrub swamp, bottom-land forest, hedgerows (?). (5691)

Stenanthium robustum Tats.--Rare; 7 ; margin of swamp on bottomland ( $597 \%$ )

Unularia sessilifolia I.-Common; $\pi$; bottom-land forest. (5029, 5089)

Dioscorea glauca Muhl.--Occasional; $\mathbb{W}$; swamp and terrace forest. $(5441)$ 
AMARYIIIDACEAE:

Erooxis hirsuta (I.) Coville.--Occasional; $\mathbb{T}$; bottom-land to upland forest. (5165)

IRIDACEAT:

Iris virginica I. (?)--Cccasional; $\mathbb{N}$, I, $S$; marsh. (5208, 5835)

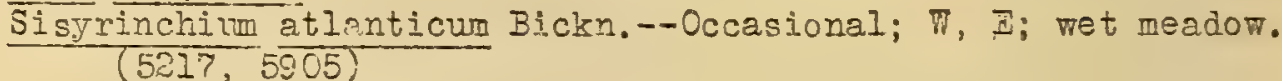

S. Elamineum Curtis,--Occasional; $\pi$; ret meador. (5192, 5878)

ORCHIDACEAE:

Gypipedium acaule Ait.--Locally common; $\pi$; slope and upland forest. (5122)

Epipactis pubescens (Milld.) A.A. Eaton.--0casional; $\pi, s$; terrace and s.lope forest. (5406)

Habenaria ciliaris (I.) R. Br.--Rare; E; brushy wet meadow. (5375)

H. lacere (Wichx.) R. Br.--Rave; $W, S$; open margin of swamp iorest. (5971)

Pogonia verticiliata ( Filla.) Natt.--Occasional; $\mathbb{W}$; terrace and slope forest. (5121, 5548)

Spiranthes cernua (I.) Richard.--Rare; 7 ; wet meadow. (5592)

Tipularia discolor (Pursh) Iutt.--Occasional; $\pi$; terrace forest. $(5063,5403)$

\section{SAIICACEAE:}

Populus alba I.-Rare; E; brushy abanconed field. (5643)

P. Erandidentata Michx.-Occasional; $\mathbb{T}, \mathrm{S}$; margins of slope and upland forest. (5I88)

Salix humilis Marsh.--Occasional; $\mathbb{T}, E, S$; abandoned fields. (5060, 507s, and 5658, det. C. R. Bali)

S. ni gra Marsh.-Occasional; T; shrub swamp. (5447 and 5892, det. C. R. Ball)

S. tristis Ait.--Rare; $\pi, E$; abandoned fields. (5637, det. C. R.

\section{JUGIAIDACEAT:}

Carya alba (I.) K. Koch.-Occasional; $\mathbb{W}, \mathrm{S}$; upland forest. (5653)

C. cordiformis (Wang.) K. Zoch.--Rare; $\pi, \bar{s}$; bottom-land and terrace forest. (565I)

C. glabra (Mill.) Spach.--Rare (?); $\pi$; bottom-land and terrace forest. ( $5613,5697,5700-$ pubescent form)

Juglans cinerea I.--One tree; $F$; bottom-lend forest. (5685)

BETTIACEAD:

Alnus rugosa (DuRoi) Spreng.--Common; $\pi, 5$; shrub smamp. (5588)

Betula nigra I.-Abundant; $\mathbb{T}, \mathbb{I}, S$; swamp and bottom-land forest. (5647)

Cerpinus caroliniana Talt.--Common; $\pi$; bottom-land forest. (5170) Corylus americana Filt.-.Occasional; $\pi$; well-drained bottom-land forest, hedgeroms and moods margins. (5440) 
FAGACEAE:

Castaner dentata (Mrrs.) Borkh.--Occasional; $\mathbb{T}$; slope and upland forest. (5239)

C. pumila. (I.) Mill.--Occasional; T, S; cut-over slope and upland forest. (5240)

Fagus grandifolia, Ehrh.-Abundant; $\pi, S$; bottom-land, terrace, and slope (occasional) forest. (5081)

quercus alba I.--Abundant; $\pi, 5$; bottom-land (occasiongl) to upland forest. (5696)

Q. bicolor Willd.--Occasional; $\pi, \mathrm{s}$; swamp, bottom-land and terrace forest. (5052 1/2, 5698)

Q. borealis Michx., var. maxima (Marsh.) Ashe.-Occasional; $\mathbb{T}$; bottomland to upland forest. (5761)

Q. coccinea Miruench.-Abundant; $T$, S; terrace to uplond forest. (5695)

Q. falcata Wichx.-Common; $\pi, S$; upland forest. (57IO)

Q. ilieifolia Tiang.--Rare; $\pi$; upland forest. (5261)

Q. imbricamia Michx. (?)--Two trees; S; upland forest. (5724)

Q. Iyrata Walt.-Occasional; $\pi$; swamp and bottom-land forest. (5047, 5692)

Q. marilandica Muench.--Occasional; $\mathbb{\pi}, \mathrm{S}$; upland forest. (5654)

Q. montana Tilld.--Occasional; $\pi, 5$; upland forest. (5743)

Q. palustris Wuench.-Common; $\mathbb{T}, \mathrm{s}$; swamp and bottom-land forest. (5703)

Q. phelilus I.-Common; $\pi, S$; swamp and terrace forest. (5699)

Q. prinus I.-Occasional; $\Pi$; bottom-land forest. (5678, 611I)

Q. steliata Wang.-Occasional; $I$, S; upland forest. (5642)

Q. velutina Iam-Comon; $\pi, s$; bottom-land (rare) to upland forest. $(5750)$

URTICACEAF:

Boehileria cylindrica ( $($. $)$ Sw.-Common; $7, \mathbb{E}$; bottom-land forest. (5352)

Funulus Iupulus I.-One plant; E; bottom-land forest. (6021)

Iaportea canadensis (I.) Gaud.-Abundant; $\mathbb{W}(?)$, F. (5351)

Morus rubra I.--Rare; $\bar{T}, \mathbb{E}$; bottom-land forest (one tree) and hedgerom. (5160)

Pilea pumila (I.) A. Gray.-Occasional; W; botton-land forest. (5452)

Ülmus americana I.--Occnsional; T, $\mathrm{E}(\mathrm{l})$; bottom-land forest. (567\%, $5684,6020 ?$ )

\section{SANTALACEAE:}

Comandra umbellata (I.) Nutt.-Occasional; $W$; margin of woods on sandy terrace. (5204)

ARISTOLOCFIACEAE:

Asaruri cenadense I.--Iocally comon; $\pi$; bottom-land forest. (5115)

POLYGOINACEAE:

Polygonum arifolium I.--Conmon; T, E, S; marsh. (5674)

P. aviculare I.--Rare (?); $\pi$; abandoned field. (5705)

P. dumetorum I,--Rare; $\pi$; open terrace forest. (5598)

P. erectum I.--Rare; T; yard. (5712) 
POIYGOI:ACEAE-COntinued.

P. hydropiper I.-Occasional (?); E; roadside ditch. (5379)

P. indropiperoides Michx.--Coninon; T; march. (5323)

P. Orientale I.--One plant; S; cleared swamp. (6093)

P. pensylvanicum I.-Cormon; $\mathbb{T}, \mathrm{E}$; wet meadow and abandoned fields. $(5026,5536,5763)$

P. persicaria I.--Occasional; $\mathbb{T}$; abandoned fields. (5457, 5530)

P. princtatum EIJ.-Common; $W, \mathbb{E}, \mathrm{S}$; marsh and wet meadow. $(5469,5544$, 5752)

P. scgittatum I.--Abundant; $\pi, \mathbb{E}, \mathbf{S}$; marsh, (5545)

P. scandens I.-Occrsional; T; nargin of botton-land forest. (5543?, 6080)

P. virginianum I.-Common; $W, \pm$; bottom-lend forest. (5439)

Runex acetosella I.-Comon; $\mathbf{W}, \mathrm{E}$; abmanned fields. (5128)

R. obtusifolius I--Rare; E; Jard. (5384, 5730)

CHENOPODIACIAE:

Chenopoalum album I.--Iocally comon; T; yard. (5708)

C. amorosioides I.--Rare; W; yard. (5508)

AMARANTHACEAIE:

Amaranthus hybridus I.--Occasional; $\pi$; abandoned fields. (5720)

PHYTOLACCACEIE:

Phytolocca americana I.--Occasional: $\pi$; woods margins. (5313)

IILEC BBRACEIT:

Scleranthus annưs I.--Occasional; T; abondoned field. (5124)

AIZOACEAE:

MolIugo verticillate I.-OccasionaI; T, I, S; recentIy bared soil of abandoned fields, roadsides and clearings. (5467)

\section{CARYOPHYLLACEA}

Arenaria serpyliffolia I.-Occnsional; T; obendoned field. (5209)

Cerastiun viscosum I.-Occasionnl; T; abandoned field. (5095)

Dianthus armerio I. - Occosional; $\mathbb{T}$; abandoned fields. (5158)

Iychnis alba Mill. -Rare; T; anndoned field on sandy terrace. (5106)

Silene stellata (I.) Ait. f. -Occasional; $\pi$; open terrace forest. (5430)

Stellaria longifolia Miuhl.-Common; $\pi$; bottom-land forest. (5168)

S. media (I.) Crrill.--Iocally common; $\pi$; abandoned field. (572I)

S. proera Michx.-Occasional; T, I; bottom-land forest. (5095)

PORTULACACEIE:

Claytonia virginica I.--Very abundant; $\Pi, E$; bottom-land forest. (5083)

NYMPHAEICENE:

Muphar advena (Ait.) Ait. f.--Common; $7, \mathrm{~S}$; marsh. (5129, 5906, 6031, 6083) 
RANUNICULACEAE:

Anemone quinguefolia I.--Rare; $\pi$; bottom-land and terrace forest. (5078)

Anemonella thalictroides (i.) Spach.--Occasional; T, $\mathbb{T}$; bottom-land forest. (511.4)

Clematis Virginiana I. - Fiare; $\pi$; margins of botton-land and terrace forest. $(5680,6024)$

Ranunculus abortivus I.--Occasional; $W$; bottom-land forest. (5084)

R. acris I.-Rare; $\mathbb{E}$; abandoned. field. (5842)

ㄱ. Duloosus I.--IocnIIt common; W; abandoned field. (5127)

R. hispidus $M_{i} c h x$. (?)-Oosasional; $\pi_{\text {; }}$ runs in bottom-land forest. (5088)

R. Ioxicallis (T. \& G.) Darby-Dccasional; T; marsh. (5229)

R. pusilius Poir,--Locally cormon; $\Pi$; marsh. (5077)

R. recurvatus Poir.--Occasionel; T; boutorin-land forest. (5101)

R. repens I.--One patch; $\mathbb{B}$; marsh. (5841)

Thalictrum polygamum Muhl.-Occasional; W.S; marsh and clearing in swamp forest. (5287, 5976)

MAGNOLIACDAE:

Iiriodendron tuIipifera I.-Occasional;, S (rare); bottom-land and terrace forest. (5613)

Mognolie virginiena I.-Cormon; $W, \quad H, S ;$ shrub swamp and swamp forest. $(5645)$

ANONACEAE:

Asimina triloba Dunal.--Occasional; $\mathbb{T}, \mathrm{E}$; bottom-land forest. (5180)

BERBERIDACEAE:

PodophyIIum peltatum I.-Common; $\pi, E$; bottom-land forest. (50SI)

IAURACEAE:

Iindera aestivalis ( I.) Bl. (Benzoin aestivale).-Occasional; $T$; botton-land forest. (5172, 5607$)$

Sassafras officinale Nees \& Iberm.--Iocally common; i; abandoned fields. (5099)

PAPAVIRACEAT:

Chelidonium majus I.--Rare; $\Xi$; yard. (5845)

Sanzuinaria canadensis I.--Rare; $\mathbb{E}$; bottom-land forest. (See p.10)

FUMARI ICELIT:

Corydalis flavula (Raf.) DC.--Rare; $\mathbb{E}$; bottorn-land forest. (5816)

Dicentre cucullaria (I.) Bernh.-Occasional; $\mathbb{W}, E$; bottom-land forest. $(5814)$

CRUC IFIRAT:

Barbarea verna (Mill.) Asch.--Occasional; W; abandoned fields. (5107, 5818)

B. vulgaris R. Br.--Rare; W; yard. (5828) 
CRUC IFTRAZ--Continued

Cupsalia bursa-pastonis (I.) Medic.--Occasional; T; abandoned

fields. (E140)

Cardamine bulbosa (Schreh.) B. S. F. -Occasional; W; bottom-land forest. $(5170)$

c. pennsylvanica ibal. --Rare; warsh in bottom-1 and run. (5182)

Dentaria laciniata ianh.-Occasional; $\pi, \Xi$; bottom-land forest. $(5176,5813)$

Draba verna I.--Iocally conmon; $\mathbb{T}, \Xi$; abandoned fields, (5808)

Iepidiur cempestre (I.) R. Br.-Occasional (?); $\pi$; abandoned field. (5210)

I. virginicuta I.--Occasional (?); $\nabla$; abantoned field. (521I)

Sisytibrium altissimum I. - - Fare; $\mathrm{E}$; vard. (5849)

S. officineIe (I.) Scop., var. Ieiocarpum DC.--Rere; $\mathbb{E}$; yard. (5844)

S. thaljenure (I.) J. Gay.--Iocaliy comon; w; abandoned field on sand̄y terrace. (5071)

PODOSTDVIACEAE:

Podosteruum ceratophyllum kichr.--Locally comon; $\pi, \mathbb{E}$; river. (5688)

CRASSULACEAT:

Penthorum sedoides I. --Rare; M; nassh. (5448)

SAXIFRAGACEAE:

Feuchera arnericana I.-Cccastonal; $\pi ;$ bottom-land and terrace forest. (5186)

Eydrengea aroorescens I.--Rare; $\pi$; Terrace forest. (5686)

Itea virginice I.--Rare; $\pi$; swamp forest. (5899)

HAMAMVIIDACIAE:

Iiquidombar styraciflua I.-Abundant; $\pi, S$; wet meadow, swamp forest and bottom-1and forest. (5704)

PLATANACHET:

Platanus occidentalis I.--Oscasional; $\pi$; bottom-land forest. (5681)

ROSACEAE:

Agrimonia moIlis (T. \& G.) Britton.--Rare; T; hedgerow. (5780)

A. parviflora Ait.-Rare; $\pi$; margin of bottom-land forest. (5494)

Anelanchier oblongifolia ( $T$. \& G.) Roem.--Occasional; $\mathbb{W}$; shrub swamp. $(5074,5258)$

Aronia arbutiforia (I.) II.1.-Occasional; $\mathbb{W}, \mathrm{S}$; shrub stamp and hedgerows ( 5097 ?)

A. prunifolia (Marsh.) Rehd. (A . aroutifolia var. atropurpurea).-Rare; S; shrub swamp. (5792)

Crataesus streminea Beadle (?).--Rare; $\pi$; former clearing. (5661)

Tracaria virsiniana Duchesne.--Occasional; $\mathbb{T}$; abanāoned fields. (5100)

Geum crnadense Jacq. - Occasional; $\mathbb{E}$; bottom-1 and forest. (5347)

Potentilla canadensis I. (P. pumila).-Common; $\mathbb{T}, \mathbb{E}$; abandoned

fields on sendy terraces. (5I08) 
ROSACEAE---Continued.

P. monspeliengis I.--Occasional; $\mathbb{W}$; wet meadow. (5314)

P. recta I.--iare; $\mathbb{W}_{\text {; }}$ abandoned fields. (5896)

P. simplex Michr. (P. Canadensis of Gray's Munual).-Occasional; $\mathbb{\pi}$; ajandoned field. (5117)

Prmuns serotina Ehrh.--Occasional; $\mathbb{W}$; hedgerows and woods margins. (5189)

Rosa carolina I.--Occasional; W; hedgerows and woods margins. (5783, 5868)

R. Fanlustris Marsh.-Comon; W, S; shrub swamp. (5064, 5279, 5974)

Prubus centrais Bailey.-Rare (?); W; abundoned field. This or other devberry species common W, S. (5264, det. I. H. Bailey)

R. cuneifolius Pursh.--Rare; S; abandoned fields. (5787)

R. hisnidus I.--Abundant; $\Pi, S$; swarp forest. (5249)

R. occidentalis I.--Occesional; $\pi, E$; hedgerows and moods margins. (5425)

R. ostryifolius Rydb.-Occesional; $\pi$; former clearing. This or other highbush blackberry species common 7 , S. (5312, det. I. H. Bailey)

Sanguisorba cennoensis I.--Rnre; I, S; open shrub swamp. (5736) Spiraen lntifolia (Ait.) Borcki.-Occasional; $\mathbb{E}, S$; shrub swamp. $(5392)$

S. tomentosa I.-Occasional; $\mathbb{W}$; wet meadow. (5059, 5341)

IEGURINOSA.P:

Arnhicarpa bracteata (I.) Fern.--Comon; $\pi$; bottom-land forest. (5486)

Apios tuberosa. Moench.--Occasional; T; wet meadow. (5413, 5985)

Baptisia tinctoria (I.) R. Br.--Occasional; $\pi, E, S$; terrace to upland forest. (5390)

Cassia fasciculata Michx.--Common (?); $\mathbb{E}$; abendoned field. (5363)

C. nictitans I.-Common; $T, \mathbb{E}, \mathrm{S}$; abandoned fields. (5338)

Crotalaria sagittalis I.-Occasional; $\pi$, S; abandoned fields. (5334, $5414)$

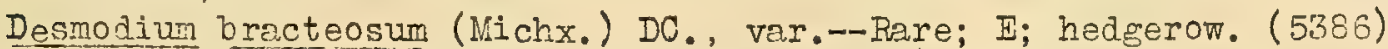

D. dilleni Darl.-Oaccusional; $\pi$; open terrace forest, abandoned

fields and hedgerows. (5578, 5599, 5617)

D. marilandicur (I.) DC.--Rare; T; Ebandoned field on sandy terrace. (5550)

D. nudiflorum (I.) DO.--Occasional; $\mathbb{W}$; terrace and slope forest. (5435)

D. obtusum (Muhl.) DC.--Occasional; $\mathbb{T}, \mathbb{E}$; sandy abandoned fields. $(5616,5633)$

D. paniculatum (I.) DC.-Occasional; T; open forest and abandoned fiela on sandy terrace. (5600,5614)

D. rotundifolium (Michx.) DC.-Dere; T; slope forest. (5877)

D. viridiflorum (I.) Beck.--Rare (?); $\rightarrow, \mathbb{E}$; abandoned fields on sandy terraces.- $(5615,5738)$

Iespedez canitata Michx.-Occrsional; I; abridoned field. (5632)

I. hirta (I.) Hornem.--Occasional; ;; abandoned field on snndy terrace. $(5611,6016)$ 
IEGUMIIVSAE--Contimed.

I. intermedia (Tats.) Britton (I. frutescens).--Rare (?); $\mathbb{T}$; abandoned field on sandy terrace. (5620)

I. repens (I.) Bart.--Occasional; $\pi$; aondoned fields on sandy terraces. (5538, 5619,5621, 5622)

I. Striata (Tinuno.) E. \& A. -Common; $\mathbb{T}, E$; abandoneả fields. (5769)

I. Virginica (I.) Britton.--Occasional; $\mathbb{T}, . \mathbb{E}$; sandy abandoned fields. $(5618,5635,5640)$

Medicago lupulina I.-Occasional; $\pi, \Xi ;$ abandoned fields. (5825)

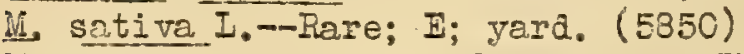

Melilotus alba Desr.-Occasional; W, I; abandoned fielós. (5846)

M. officinalis (I.) Iar.--Rare; $\pi$, $\$$; trards. (5847)

Fobinia pseudo-acacia I.-Cccasional; $\pi$; abandoned fields and upland forest. (5577)

Strophostyles umbellata (Vurl.) Britton.--Cccasional; $\mathbb{T}$; abandoned fields on sandy terrace. (5 109$)$

Stylosathes biflora (I.) B.S. P.--Rare; W; open terrace forest. $(5401)$

Tephrosia virsiniena (I.) Fers.--occasional; $\pi$; upland forest. (5238)

Trisoling agrarium I. - Locally common; $\pi$; abandoned fields. (5218)

T. arvense I.-Occasional; $\mathbb{T}, \mathbb{Z}$; abandoned fields. (5224)

T. dubium Sibth.-Occasional; $\pi$; abandoned field. (5206)

T. hybridun I.-Iocally common; 7, $\Xi$; avandoned fields. (550\%, 5831)

T. pratense I.--Occasional; $\pi, \mathbb{B}$; abondoned fields. (5282)

I. procurneens I.-Common; T. I (i); abandoned fields. (5157)

T. repens I.-Occasional; $\mathbb{E}$; abandonea field. (5832)

Vicia angustifolia Reichard.--Rare; T; abendoned field. (5879)

V. villosa Roth.--Rare; $\mathrm{E}$; abendoned. field. (5829)

IINACIAE:

Iinum floridanum (Planch.) Trel.--Rare (?); T; wet meadow (5299, 5986)

I. Striaturu Malt.--Occasional; $\pi$; wet meadows. (5987, 5991, 6100)

I. Virginianum I.--Rare; $\pi$; roadside in upland forest. (5667)

OXATIDACZAE:

Oralis stricta I.--Occasional; $\pi$; abandoned fields. (5191)

GRRANIACEAE:

Erodium cicutarium (I.) Ister.--Occasional; $\pi$; abendoned field on sandy terrace. (5070)

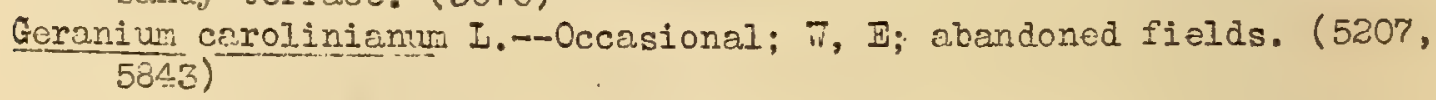

G. maculatum I.-Occasional; T; well-drained bottom-land forest.

POLYGAIACEAT:

Polygala curtissii A. Gray.-Common; $\mathbb{T}(?) ; \mathrm{T}, \mathrm{S}$; abandoned fields.

$(5332,53 \% 4,5636)$

P. Sanguinea I.-Occasional; $\mathbb{T}, \mathbb{I}$; wet meador. (5285, 5373)

P. verticillata I.--Occasional; $\pi$; abandoned field. (5324) 
ETPEORBIACEAT:

Acaljpha gracilens A. Graj.--Oceasional; $\mathbb{W}, \Xi$; partly cleared forest and abnunec fieles. (5585)

A. virginica I.-Comon; $\nabla$; clearings in bottom-land forest and abandoned fields on sanijy terrices. (5537)

Eupioroia corolinta I. - Vocasional; $\mathbb{E}$; abardonea field on sandy terrace. (5359)

E. ipecaclanhhae I.--Rare; s; clearing in upland forest. (5141)

E. presili Guss.-Occasional; $T$; abrndoned fields. (5460)

OALIITRICEAGZAP:

Celititriche austini Engelm.-Occasional; $\pi, \mathrm{s}$; demp soil in terrace forest. (52:3, 5973)

C. heteronhy?ia Fursh.--Common; $T, \mathbb{E}$; runs in bottom-land forest, springs and brooks. (523:4, 5910)

ANACARDIACEAE:

Rhus coralline I,-Comon; $\mathbb{T}, \mathbb{E}$; abandoned fields and hedgerows. $(60 \overline{6}, 5.41)$

R. Ilahra I.-Cocasional; $\Pi, E$; abardoned fields and hedgerows. $(55.40)$

R. tyinina Torner.-Occastonal; i; hedjerows. (5539)

Toxicoändron radicans (I, ) Kuntze.-Abundent; 7 ; bottom-land forest, hedgeroms, etc. 5751 )

T. vernix (L.) Euntze.-Dommon; T, E, S; shrub swamp. (5393)

AQUIFOLIACEAE:

Ilex laevigata (Pursh) A. Gray.--Rire; S; swamp forest. (5791)

I. opaca Ait.-Comon; $\bar{W}, S$; terrace and slope forest, and hedgerots. $(5068,5153)$ I. Terticillata (I.) A. Gray.--Dominon; $\pi, E, S$; shrub swamp and swamp

CEIASTRAOEAE:

Evonjmus arericanus I.--Occasional; 7 ; bottom-land forest. (5167)

ACIRACEAT:

Acer rubrum I.-Conmon; $T, S$; swamp forest and bottom-land to upm land forest. (5655)

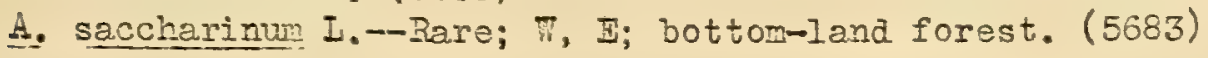

BAISAMINACELE:

Impotions biflora Talt.--Occasional; ; bottom-land forest. (5288)

RHAMIACEAT:

Ceanothus americanus I.--Rare; "; upland forest. (5912)

VITACTAE:

Parthenocissus quinquefolia (I.) Planch.--Occasional; ; bottomland forest and heagerows (?). (5690) 
VITACEAB--Continued.

Vitis aestivalis Michx,-Occasional; $\mathbb{T}, \mathbb{F}$; hedserows and roods mergins. (5030, 5222, 5639)

V. cordifolja Iam.-Occasional; $T$; Dottom-land forest and hedgerow. $(5169,5682)$

V. labrusca I.--0ccasional; $T$; heăkerors and wargins of bottom-land and terrace forest. (5156, 56:1;

\section{MALVACIAE:}

Abutilon theophrasti Weoilc.--Iocally common; ; abandoned field. (5364)

Malva neglecta Tallr. -Rere; 7 ; yard. (5506)

Sida spinosa, I. --Rare; E; abandoned rieid. (5365)

EYP HPICACFLE:

Ascyrum hypericoides I.--Occasional; T; terrace and upland (?) forest and avandoned fields. (54:02) Hipericuri canadense I.--Occasional; 7 , S; wet meadow. (5293, 5421,

H. denticujatur WaIt. (H. virisatum)--Iocally common; $\mathbb{W}, \mathbb{E}$; abandoned fieids on sandy terraces. (5743, 5989)

H. gentianoides (I.) D. S. F.-Common; T; abandoned fields (?). (5422)

H. mutilum I.-Coinnon; 7 ; mersh. (5325)

H. perforatum I.-Occasional; $\mathrm{H}, \mathrm{B}, \mathrm{S}$; abandoned fields. (5913)

H. petiolaturn Nalt.--Rare; T; morsh. (5916, 6014)

I. puncteturi Iam.--OccasionaI; $(5300,5450)$

H. Virginicum I.-Cccasion?I; $\pi, \mathbb{Z}, \mathbb{S}$; wersh. (5483)

CISTACAㅍ:

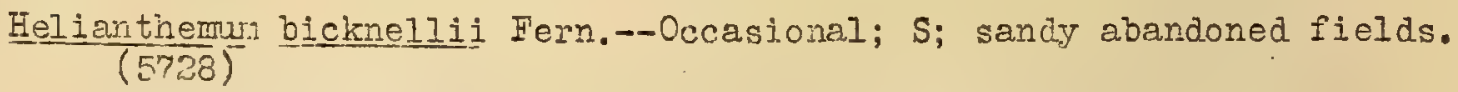

H. canadense (I.) Michx.--Rare; T, S; open upland forest. (5711,

Lechea. racemulosa Michx.--Common; $\pi$; abandoned fields on sandy terrace. $(5610)$

VIOIACEAE:

Viola affinis IeConte.-Common; $\pi, \mathbb{E}$; bottom-land forest. (5086, 5815)

V. britioniana Pollard.--Rare; $\pi$; wel.l-drained, open bottom-land forest. (60II)

V. cuculiata Ait.-Occasional; $\pi$; marsh. (5076, 5820)

V. eriocerva Schrein.-Occasional; i7; oottom-land forest. (5178, 5819)

$\overline{\mathrm{V}}$. lanceolata I.--Iocally common; $\mathbb{T}$, S; marsh and wet meadow. (5072)

$\vec{V}$. primulifolia I.-Occrsional; $\nabla, \mathrm{S}$; wet neadow. (5073)

$\bar{V}$. sacitata Ait.--Occasional; $\pi$, $E$; wet meadow. (5092)

IYTHFACEAI:

Cuphea petiolata (I.) Koehne.--Occasionnl; W; abandoned fields (5465)

Rotala ranosior (I.) Toehne.-Occasional; $\pi$; marsh. (5482, 5717, 6025) 
MELASTOMACEA :

Rhexia meriena I.--Common; W, D; wet meadow. (5275)

R. VIIginica I. - Occasional; T; wet meador. (5II7)

ONAGRACHIS:

Circuea 1atifolia Hill.-Occasional; ; bottow-land forest. (5308)

Eoilobium coloratum Muht.-Occasional; $W$; wet meadow. (5475)

Iudvicic alterifolia I.-Occasional; W; T.et meadow. (5319)

I. pajustris (I.) EII.-Abundant; T; marsi. (523?)

Genothern biennis I.-Occasional (?); T; abandoned field. (5542)

- $\quad$. Inearis Michx.-Occasional; W, S (?); abandoned fields. (5289)

O. Iorinediceliata (Small) Robinson.-Cominon; $\pi$, $ᄑ$; wet merdor. $(5215,5904 ?)$

FAIORAGIDACEAJ:

Proserpinaca palustris I.--Occasional; $\mathbb{N}, \mathbb{\Xi}$; marsh. (5356, 6102)

ARAIIACEAD:

Aralia nudicenl is I.--Occasional; $\pi$; terrace forest. (5446, 5822)

A. spinosa I. - Rare; S; terrace forest. (6112)

Panax trifolium I.-Occasional; W, E; bottom-land and terrace forest. $(5080)$

\section{UNBEIIIFHRA.}

Angelica, villosa (Talt.) B. S. P.--Pare; 7 ; upland forest. (5784)

Cicuta raculata I.-Occasional; it; marsh. (532a)

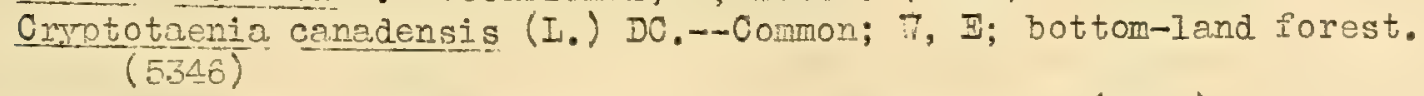

Daucus carota I.--Occasional, F; abandoned fields. (5325)

Eycrocotele emericena I.--Rare; 7; bottom-land forest. (5306)

Otpolis igior (I.) Coult. \& Rose.-Occasional; S; marsh. (5024, $(5, ; 7)$

Sanicila conejonsis I. --Pare; T; bottom-land forest. (5309)

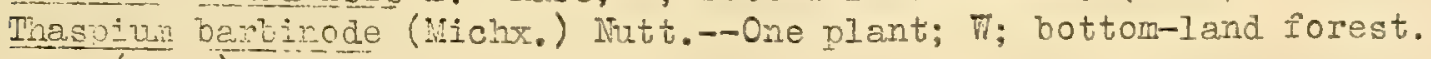
$(6110)$

CCRNACÐAE:

Cornus amomum Will.--Occasional; $\pi$; 푸 margins of bottom-land forest. $(5496,5644)$

C. florica I.--Common; T; bottom-Iana to unoland forest. (5I03)

Nyssa sylvatica Marsh.--Occasional; T, S; swamp forest. (5278)

FRICACEAF:

Chimaphila maculata (I.) Fursh.--Common; $\pi$, s; terrace and slope and upland forest. (5305)

C. umbeliata ( $\left.I_{3}\right)$ Bart.-Occasional; $\pi$ (rare), S; slope and upland forest. (5575)

Clethra alnifolia I.--Common; $\mathrm{S}$; shrub swamp and hedgerows and woods margins. (5545)

Epigaea repens I.-Occasional; $\pi, s$; terrace to upland forest. (5625) 
ERICACEA -Continued.

Gemltheria procumbens I.--Iocally common; W; terrace to upland forest.

Gaylussacia haccata (Tang.) K. Koch.--Abundant; W, S; slope and unleind forest. $(5120)$

G. frondosa (I.) T. \& G.--Common; M, S; swamp forest (occasional) and slope and upland forest. (5195, 5393)

Kalmij, aneustifolia I.-Occasional; W, S; swamp forest. (5196)

K. Iatifolia I.--vommon; Th, S; terrace to upland forest. (5147)

Ieucothoe racenosa (L.) A. Gray.-Common; W, S; swam forest. (5123)

Iyonia ligustrina (I.) DC,-Conmon; W, S; swamp forest, brushy wet meadows, and hedgerows. (5296)

I. mariana (I.) D. Dor1.-Cccasional; T, E, S; terrace to upland. forest. (5146)

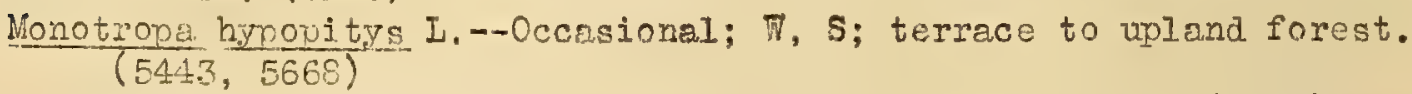

M. uniflora I.--Occasional; $T$; terrace and slope forest. (5250)

Pyrola americana Sweet.-Common; $W$; terrace and slope forest. $(5061,5330)$

P. chlorantia Sw. (?)--Rare; s; upland forest. (5062)

P. elIiptica Nutt.--One patch; s; uplend forest. (5789)

P. secundel I. - Pare; S; slope forest. (5804)

Rhododendron nudiflorum (I.) Torr.-Occasional; $T, s$; terrace to upland forest. (510\%)

R. Viscosum (I.) Torr.-Occasional; T, S; shrub swemp. (5194)

Vaccinium atrococcum (A. Groy) Eeller.--Occasional; it, $\mathbb{E}, \mathrm{S}$; shrub swamp, abandoned fields and. hedgerows. (5075, 5925)

V. Stanineum I.--Occasional; S; slope and upland forest. (5142)

V. vacillans Kalm.--Abundant; $W, S$; slope and upland forest and abandoned fields. ( 5119 and 5926 , both var. crinitum Fern.)

\section{PRIMUIACEAE:}

Anagallis ervensis I.--Rare; S; recently broken soil in orchard. (5915)

Iysianchie nuinularia I.-Common; $W$, $\mathrm{F}$; bottom-land forest. (5407)

I. producta (A. Gray) Fern.-Iocally common; T; abendoned field on bottom-land. (5292)

I. quadrifolia I.-Occasional; $\mathbb{W}$; terrace to upland forest. (5241)

I. terrestris (I.) B. S. P.-Locally common; W; wet meadow. (5295)

Steiruncma ciliatum (I.) Raf.-Comon; 7 (?), E; bottom-land forest. (5355)

S. Ianceolatum (Walt.) A. Gray.--Occasional; W; wet meadow. (5320 $5990)$

\section{EBENACFAE:}

Diospyros virginiana I.--Cccasional; $\mathbb{W}$; bottom-land forest, abandoned fields and hedgerows. (5223)

OIEACEAE:

Chionanthus virginica I.--Occasional; $W, E$; bottom-land and terrace forest. $(5162,5826)$

Fraxinus biltmoreana Beadle.-Occasional; $T$; bottom-land forest. $(50 \pm 5,5687 ?)$

F. Dens.ylvenica Marsh. (?)-Occasional; W; bottom-land forest. 
GENTIANACEAF:

Bartonia paniculate (Michx.) Robinson.-- Hame; (5707)

Gentinn clausa Rnf. (?)-Occasional; W, I, S; wet meadow. (5725, 6]. 03 1/2)

Sabatio angularis (I.) Pursh.-Occasional; $\pi$; abailoned fields on sondy terrece. (5410)

APOCYNACEAT:

Apocynum cannabinum I., var. pubescens (R. Br.) DC.--Occasional; $\pi$; wet meadow. (5396)

\section{ASCI HPP IADACBA}

Acerates viridiflora $\$ 1$ I.--Rare; $\pi, S$; abandoned fields. (5970) Asclepias amplexicavis Sm.--Rare; T; abandoned field. (5900)

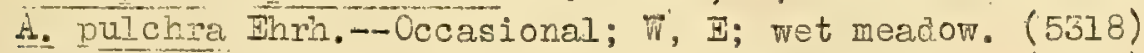

A. syriaca I.-Occasional; $\pi$; abandoned fields. (5454)

A. tuberosa I.--Fare; $\%, S$; abondoned fields. (5301)

CONVOLTULACEAT:

Convolvulis sepium I.--Rare; $\pi, \mathbb{E}$; abondoned fields.. (5903, 5984)

Cuscuta compacta Juss.--Rare; $\mathbb{W}$; woods margin. This or other species occasional N, S. (5416)

Ipomoea hederacea Tacq.-Rare (?); F; abandoned fields. (5525)

I. Iacunosa I.-Occasional; 7 ; abananed fields. (5526)

I. pandunata (I.) G. F. W. Mey.-Occasional; W, $I$; abandoned fields on sendy terraces. (5361, 5551)

I. Purpurea (I.) Roth.--Rare; W, I; yards. (5456)

POIEIMNIACEAT:

Phlox maculata I.--Occasional; T; open forest and hedgerows and woods margins on sandy terraces. (5344, 5424)

HYDROPGYLIACENE:

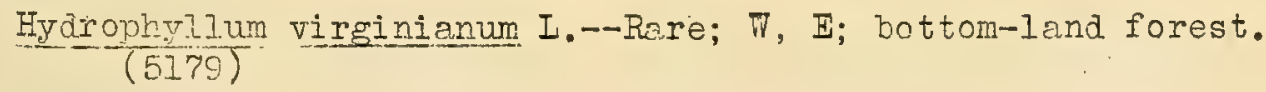

BORAGINASEAE:

Mertensia virginica (I.) Iink.--Occasional; $\Pi, E$; wottom-lnnd sorest. $(5113,5817)$

Myosotis laxa Lehm.-Occasional; $\pi$; marsi. (5231, 5230)

VERBEIACEAT:

Verbena hastata I.--Locally common; W, $\mathbb{E}, \mathrm{S}$; wet meadow. (5370)

Verbena urticepiolia I.-Occrsionel (?); E; abondoned field. $(5382)$

\section{IABIAMAE:}

Collinsonia canadensis I.--Locally common; $\pi, E$; bottom-land and terrace forest. (5431)

Cunila origanoides (I.) Britton.--Rare; s; upland forest. (5802, 6096) 
IABIATAT-Continued.

Hedeorn pulegioides (I.) Fers.--OccasioneI; T; open terrace and upIand forest. (.5493)

Lamiun amplexiccule I. - Rare; $\pi$; yard. (5236)

Leomurus cardiaca I. --Rare; $\mathbb{E}$; yaxd. (5830)

Iycopus nuericnnus Muhl.-Occasjonel (?), E; met meadow. (5378)

I. uniflorus Michx.--Iocally comon; S; cleared shrub swamp. (5726)

I. vinginicus I. - Occasional. (?); Ni; narsh. (5399)

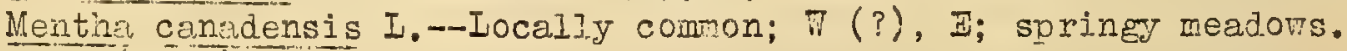
(537)

Nepeta hederace (I.) Trevisan.--Locally common; $\pi$; former clearing. $(5125)$

Perilia frutescens (I.) Britton.--Rare; $\mathbb{T}, \mathbb{E}$; open bottom-land forest. (5566)

Prunella vulgaris I.-Occasional; W, $\mathbb{E}$; abandoned fields. (538I)

Pycnanthemum flexuosum (Walt.) B. S. P.--Occasional; $\pi$; wet meadow. $(5342)$

P. incanury (I.) Michx.--Rare; 7 ; clearings in terrace and slope forest. $(5436,5779)$

P. muticum (Michx.) Pers.-Occasional; T, I; wet meadows and abandoned îeld. (5343, 5732)

P. vericillatur (ifichx.) Pers.--Rare; $\nabla$; clearings in terrace and slope forest. ( 5437,5778$)$

Scutellaria integrifolia. I.--Occasional; F; ret meador. (5294)

S. lateriflora I. - Occasional; $\pi, S$; morsh. (5449)

S. ovalifolic Pers.--Rare; $\pi$; open terrnce forest. (5891, 5911)

Teucrium candense I.--Rare; I; bottom-Irna forest. (5348)

Trichostema dichotomuja I.-Cominon; $\mathbb{T}$; ajandoned fields. (5058, 5527, $5528)$

\section{SOIAITACEAE:}

Datura stramonium I.--Occasional; E; yard. (573I)

Physalis subglabrata Mack. \& Bush.--Rnre; 7 ; abondoned field and vard. (5781, 6101)

Solanum carolinense I.-Common; $\pi$, $\mathbb{E}$; abandoned fields. (5263)

S. nigruin I.-Rore; $\bar{i}, \mathbb{\Xi}$; yards. (5719)

SCROPHUIAFIA GHAE:

Aureo ra virginica (I.) Pennell.--Occasional; $\pi$; terrace and uplend forest. (5400, 5673)

Cholo lebre I.-Rare; T, S; marsh. (5572, subsp. typica)

Gerarija decemloba Greene.-Occasional; B; abandoned field. (563I)

G. Jourcurea I.-Common; $\pi, \mathbb{I}, \mathrm{S}$; rbandoned fields. (5549)

Grntiola virsiniana I. (G. sphaerocarpa).--Occesional; $\pi$; mun in bot tom-Iand forest. (5183)

Linaria conadensis (I.) Dumont.--Occasional; $\pi$; abandoned fields. (5126)

Lizdernia enagallider (Michx.) Pennell.--Occasional; $\pi, S$; marsh. $(5481,6032)$

I. dubia (I.) Pernell, subsp. major (Fursh) Pennell.--Occasional; $\pi, \mathrm{s} ;$ marsh. $(5480,5675,6033)$

Melampyrus linenre Desr., subsp. Iatifolium (Muhl.) Beauverd.-Occasional; 7 , s; terroce and slope forest. Some sight records nay not be this subspecies. (5254) 
SCFOPHUIARIACELE--Continuvd.

lijulus alatus Ait.-Conmon; $\mathbb{T}$; bottor-lond forost. (5408)

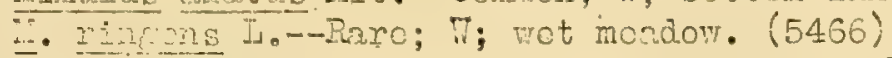

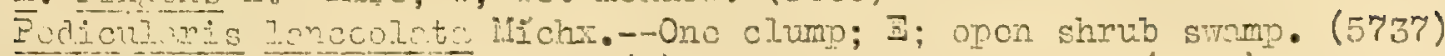

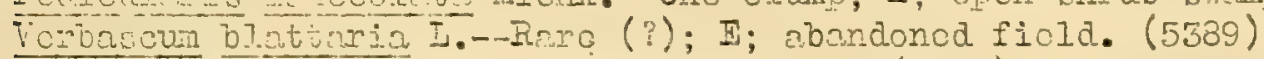

V. thapsus I.-Iiare; $\pi$; abandoned field. (5265)

Voronica arvonsis I.-Occasional; $\pi$; abnadoned fiolds. (5094, 582I)

T. Pricinails I.-Oecasionol; T,S; abandonod fiolds (5149)

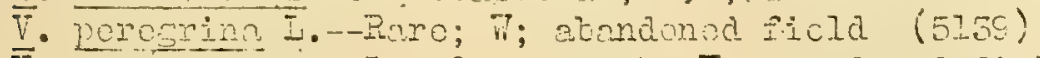

$\vec{V}$. Serpylifolia I. -occasional; $\pi$; abundonod ficla. (5093)

LENTIBUTLRINCENE:

Utricularic Eominiscana Bonj.--Raro; $\pi,, S$; brooks. (5992, 3070)

OROBAITCHACA

Epifamus virginiona (I.) Bnrt.--Comon; $\pi, S$; terrace and slope forest.

Orobanche uniflora I.--Rare; $\pi$; bottom-land forest. (5134)

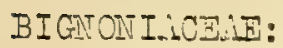

Tecoma radicnns (I.) Juss.--Iocalyy common; $\mathbb{W}, \mathbb{E}$; abandoned fields on sandy terraces. (5366)

PERYI AOZNA:

Phrymin leptostachra I.-One plant; $\Pi$; bottom-lond forest. (5453)

PIAITAGINACAIE:

Plentogo aristata lichx.--Occosional; $\Pi$; rondside. (5335)

P. Inceclata I.-Commin; T; abendoned fiolds. (5104)

P. mela Dono.-Conron; $\pi$; abnndoned fields. (5459, 5532)

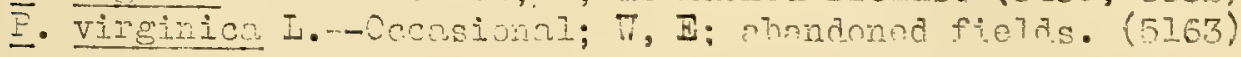

RUSTLCE:T:

Cophalanthus ocidentalis. I.--Common; W, I, S; shrub swanp. (5281)

Diodia teros Walt.-Abundnnt; $W, \mathbb{E}, S$; abnndoned fiolds (5418)

Gallum aparine I.-Common; W; bottom-land forest. (5133)

G. circoezens Michx,--Raro; $\mathbb{W}$; torrace forest. (5311, 5426)

G. Cloytoni Michx.--iocally common; W; wot moadow. (5233)

G. Dilosum Ait.--Rare; W, S; upland forest and abondoned ficld on samdy terrace. (5713, 5749)

G. tinctorium I.--Occasional; $N$; bottom-land forast. (5185, 5253).

$\vec{G}$. Eriflorum Michx.--ocensional; $\mathbb{W}, \mathbb{B}, \mathrm{S}$; bottom-land and terroce forost. (5549, 6109)

Eoustonia cremulas $I_{0}-$ Conton; $W, \mathbb{W}$; bottom-land forest, wet mendow, abandoned fielas, etc. (5082)

F. Nurpurea I.-Ooccasional; i; bottom-land forest, etc. (?) (5184) Witchellar repens I.-Coninon; $W, S$; terrace and slope forest. (5022, 5272 )

Oldenlandia uniflora I.--Rare; W; wet meadow. (5714) 


\section{C.PEIFCIIACE:AE:}

Ionicera taponica Thurb.-Comion; $\mathbb{T}, \mathbb{E}, \mathrm{S}$; bottom-land, stamp and terrace forest, abandoned fiej.ds and hedgeroms. (5154)

Sambucus canadensis I.--Occasional; it, s; bottom-land forest, shrub straxp and hedgeroms. (5221)

Viburnum acerifolinm L.-Cccasional; $\pi$; vell-drained bottom-land and terrace forest. (5164)

V. dentatuin I.-Occasional (?); $\mathbb{E}$; slirub swamp. (5834)

V. nudum I.-Occasional; T; swamp forest. (5065, 5553, 5767, 5827)

$\bar{\nabla}$. Drumifolium I.-Occasional; $\pi$; bottom-lard forest. $(5045,5112)$

$\overline{\mathrm{V}}$. pubescens (Mit.) Pursh.-Comion; $\mathbb{T}, \mathrm{S}$; bottom-land, stanp and terrace forest and hedperows. (5050, 5220, 5579, 5605, 5362, 5795)

\section{CUCUREITACENT:}

Sicyos angulatus I.--Rare; W; bottom-land forest. (5676)

\section{CANPATUTACDAE:}

Specularia perfoliata (I.) A. DC.--Occasional; $\pi, \mathbb{E}$; abandoned

\section{IOREIIAGESA:}

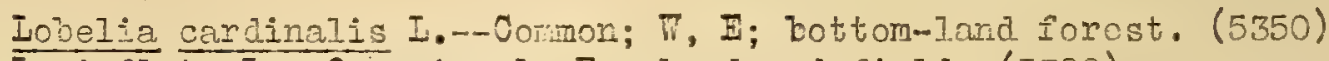

I. inflata I.-Occasional; $\Xi$; abandoned field. (5380)

I. proeruIa Iichx.--Incaliy comon; 7 ; ret merdor. (5.53, 6552)

I. Spicate Inm.--Rere; $\mathbb{W}$; open forest and boindoned ficld on sandy terreco. (541j)

COIPOSIMAIE (Determined bu S. F. Blale, excent as otherrise noted): Achillea millefcliur I.--Cccasional; $\pi$; abandoned fields. (5225) Actinomeris altemifolia (I.) DC.--Rnre; $\mathbb{T}, \mathbb{E}$; open bottom-land forest. (6022, det. Fotchiss)

Anbrosia artenisiffolia I.--Comon; $\pi, \Xi$; abandoned fielảs. (5057, $5509)$

1. trifica I.--Rare; $\pi$; oper bottom-land forest. (5492)

Antenaria nerlocta Groono.-Occasional; $\mathbb{T}, \Xi$; abandenod ficlas. (5126) (5810, det. Hotchliss)

1. neodioica Greone.-Rare; T; abandoned rield. (5118)

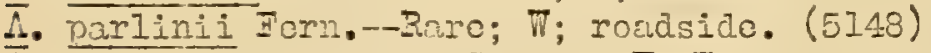

Anthomis amponsis I.--Comion; $\pi$, E; abandonod fiolds. (5205) (5895, dot. Fotchkiss)

A. cotula I.--Tare; $\Xi$; Jard. (5914, det. Hotchliss)

Irctium minus (Hill) Dernh.--Occasional; $\Xi$; yard. (5385)

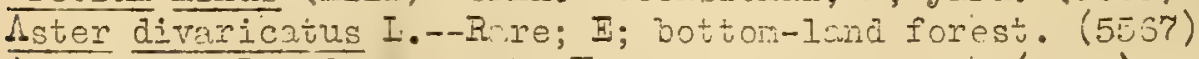

1. äjusus I.-Cocasional; $\pi$; open shrub stremp. (5554)

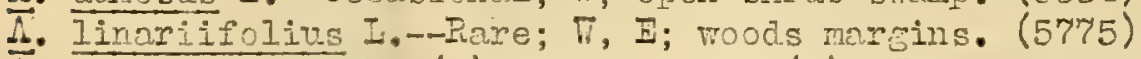

I. paniculatus Ian. (?).--OccasionaI (?); $\mathbb{R}$; open shrub swamp (5555) and roadside. (5760, form)

1. umbellatus iili..--Fare; $\pi$; wet mecdor. (6039)

I. unduratus forest. (5601, 5776)

1. Vimineus Inn.--Oecasionol; S; roandoned field. (5746) 
COMPOSITAE-COntinuod.

Eidons bivinnota I.-.Raro; E; yard. (5627)

B. coninta lifiht...-Comnon; W; marsh. (5558)

D. IJI ase I.-Hare; T; met meadow. (5534)

E. Vhite Greenc.-mine; W; abandoned field. (5yaz)

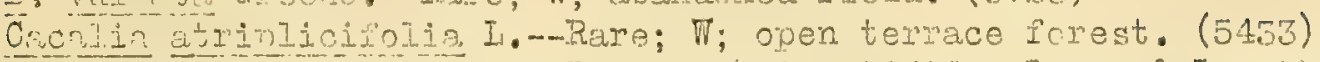

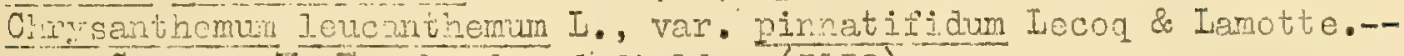
Common; $\mathbb{T}$, abandoned fields. (5152)

Chrusopsis marians (I.) întt.--Cormon; W, E, S; terrace ionent (rame) are ritucond, often brushy fields. (5654)

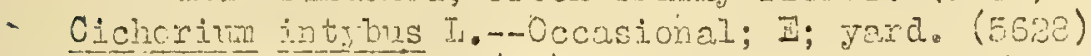

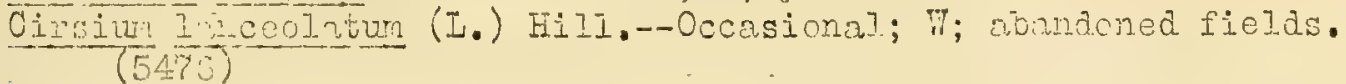

Corcopsis verticillota I.-Occasional; W, S; upland forest and abandoned fielcs. (5442)

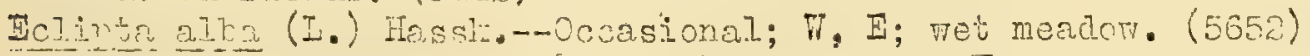

Eloriantonus canolinianus Willd.-Occasional; T; woods margins. (5557)

Erechtites niemeirolia (I.) Raf.-Comon; T, S; clemred swamp and un land (raine) forest. (5502)

Iriceron annuvs (I.) Pers.-Comon; $\mathbb{T}$, $\Xi_{\text {; }}$ abandoned fields. (5202)

E. Canadensis I,-Common; T, E; abandoned fielcis. (5461)

- ramosus (Wo,t.) B. S. P..-Gorinon; T, I; abmaned fields. (5201)

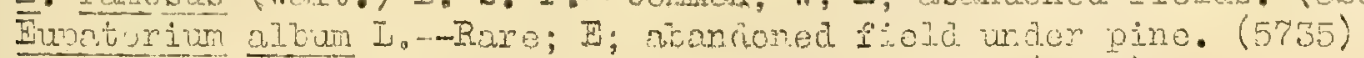

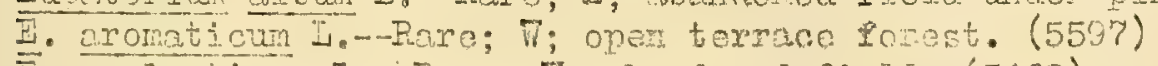

E. colestinum I.--Rare; 7 ; abmdonod field. (5462)

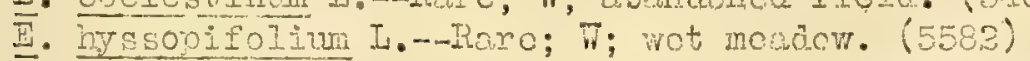

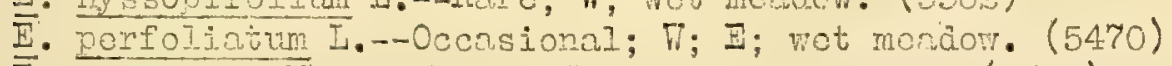

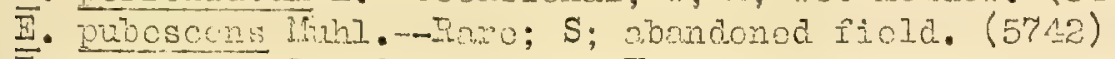

E. purpiroura I.--Occasionel; W; wot moadow and abandonod fiold.

E. rotundifolium J.--Occasional; 7 ; wot mocdow. (5590)

E. torrotonm Short.--Raro; E; cbandoncd fiold among pinos (5611)

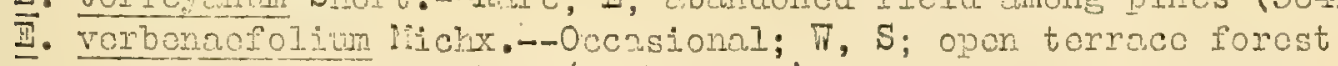
and andonod fiold. (554t, 5741)

Goincome cilia? (Raf.) Blake.-Rare; T; yard. (5235)

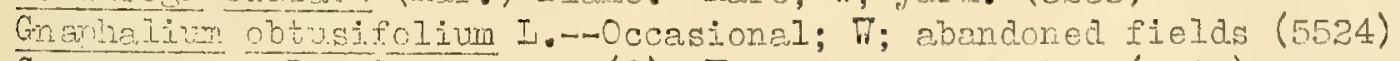

G. mineen I.-Occasional. (?); T; abandoned field. (5190)

Foleniru Entumale I.--Rare; $\pi$, E; met meadow. (6023)

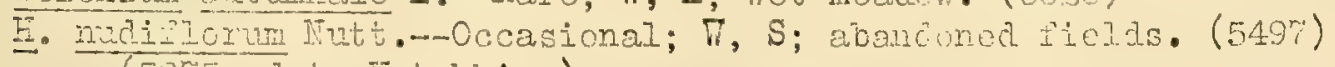
(5975, det. Hotchliss)

Eelianthus sigarteus I.-Occasional; $\pi$; rarcins of bottom-land and terrace forest. (5495--form, 5535)

Hicraciun sonoti I.-Occasional; T; abandoned field on sandy terraco. (5412)

E. paniculatura I.--Occasional; woll-drained bottom-land and terrace forost. (56,1)

H. Scabrum Michx.-.-Raro; $\pi$; woods margin. (5420)

$\overrightarrow{\mathrm{H}}$. Venosum L.--Ocossional; $\Pi$; slope and upland forost. (5242)

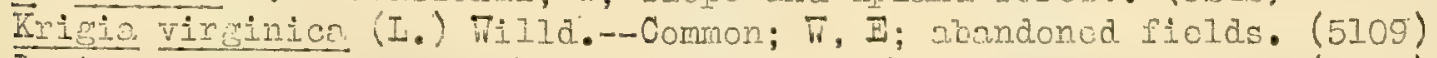

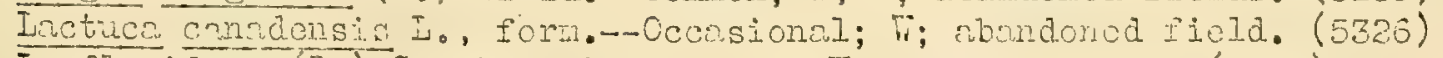
I. floridana (I.) Gucrti.--Occasional; $\Pi$; woods margins. (5415) 
COMPOSITA-J-Jontjnited.

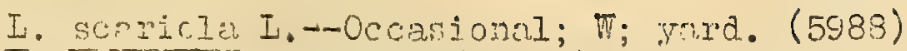

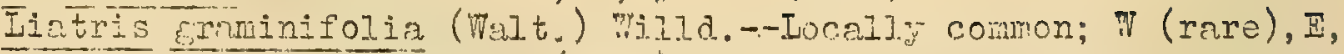

S; zbrudoned fields. $(5630)$

Mikenia scandens. (I.) Wijld.--Rnre; $\pi$; bottom-land forest. (5606)

Prencities seipenteria Tursh.-Rale; W, $\mathrm{E}$; roods margins. (5602)

(6081, det. Fotchkiss)

Fuabeclia hirte I. - Jocally common; W, s; abandonea fields. (5219)

R. Iaciniata I.-Occasionai; W, IF; pottom-land forest. (5485)

Serecio aureus I.--Rare; T; bottom-Innd forest. (5174)

S. Smaliji. Britton.--Rare; $*$, E; abandoned fields. (5901, det. Hoteh-

Sericncarpus asteroides (I.) 3. 3. P.--Occasional; S; slope forest.

S. Iinifolius (I.) B. S. P.-Comion; E, S; abandoned fields. (5333)

Solides altissima L. -Occasional; 7 ; doandoned field. (5576)

S. bicolor I.--Rare; $W, \mathrm{E}, \mathrm{S}$; well-drained bottom-land to upland forest. (5665)

S. covis. I.-Rere; "\#; onen terrace forest. (5596)

S. Eriminifolia (I.) Salisb., Var. mutnliii (Greene) Fern.--Rare; "i; andoned field. (5529)

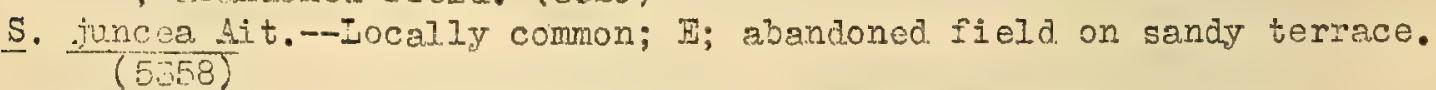

S. nernoralis Ait.--Iocally common; 7 ; abandoned field. (5589)

S. puberula Nutt.-Locally comon; 7, s; open uplend forest and abandoned field. (5666, 5727)

S. Mrosi Mili.--Occnsional; T; open terrece forest. (5432, 5546)

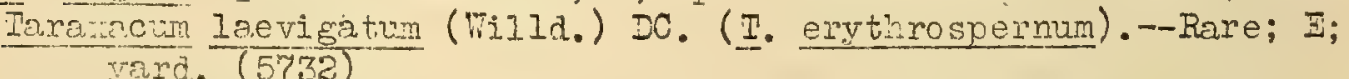

T. oificinale Teber.-Occasional; $\pi$, E; partly cleored terrace forest (rare) and yards. (5823, det. Eotchkiss)

Vernorin noveborncensis Willd.-Occasional; W; wet meodow. (5471)

Xinthium puncens vel nr.-Occasionel; $\pi$; abndoned fields. (5702) 

OK 133. How

otchkiss. Neil/Flora of the Patuxent Re

||||||||||||||||||||||||||||||||||||||||||||||||||||||||||||||||||

35185001505773 
